\title{
A topological approach to three dimensional laminar mixing
}

\author{
N. D. Jewell ${ }^{1}$
}

(Received 22 October 2009; revised 12 April 2012)

\begin{abstract}
In recent years, topological concepts have yielded valuable insights into the long standing problem of laminar fluid mixing. Topologically complex stirring protocols are typically far superior to topologically simple protocols, guaranteeing chaotic advection of fluid particles and the associated exponential dilation of material elements. Furthermore, topological approaches to mixer design are typically intuitive and insensitive to precise geometry or fluid properties. However, results to date have been limited to two dimensional flows (for example, batch stirrers in food or polymer manufacturing) and quasi three dimensional protocols (for example, continuous flow micromixers). Motivated by a simple stretching and folding argument that works well in two dimensions, we propose a topological approach to fully three dimensional fluid mixing. A transition matrix is derived to describe the mapping induced by a three dimensional 'braid' on area elements,
\end{abstract}

http://journal.austms.org.au/ojs/index.php/ANZIAMJ/article/view/2557 gives this article, (c) Austral. Mathematical Soc. 2012. Published April 20, 2012. ISSN 1446-8735. (Print two pages per sheet of paper.) Copies of this article must not be made otherwise available on the internet; instead link directly to this URL for this article. 
and the associated Perron-Frobenius eigenvalue provides a prediction of the large time asymptotic area growth rate. We show that these theoretical predictions agree well with numerical data obtained from simulations in a prototype three dimensional mixing device.

\section{Contents}

1 Introduction

C876

1.1 Non-inertial fluid mixing . . . . . . . . . . . . . C876

1.2 The $2 \mathrm{D}$ pigtail protocol . . . . . . . . . . . . C876

1.3 The 2 D clockwise protocol . . . . . . . . . . . . . C878

1.4 Braids . . . . . . . . . . . . . . . . . . C C880

1.5 Ghost rods. . . . . . . . . . . . . . . . C C883

1.6 The next frontier: from 2D to 3D . . . . . . . C C883

2 A theory of 3D mixing

C884

2.1 Advection and topology . . . . . . . . . . . . . . C884

2.2 A theory of 3D braids . . . . . . . . . . . C886

2.3 Practical issues: from braids to protocols . . . . . . . . . C891

3 Method

C893

3.1 A hypothetical batch mixer . . . . . . . . . . . C893

3.2 Surface tracking algorithm . . . . . . . . . . . . . . C899

4 Results

C900

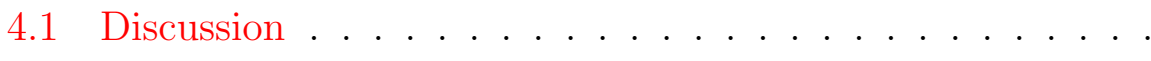

C901

References

C908 


\section{Introduction}

\subsection{Non-inertial fluid mixing}

Mixing represents an important yet relatively little studied topic in fluid mechanics. At the most fundamental level, mixing results from the (generally slow) process of molecular diffusion. In most practical applications of fluid mixing, homogenisation is greatly accelerated by inducing turbulence or unsteady advection, resulting in rapid dilation of fluid elements and chaotic Lagrangian particle trajectories. In many instances fluid mixing must be promoted in the considerably more challenging laminar regime, either because turbulence is precluded by low Reynolds number (for example, dough, molten glass, microfluidic devices $[9,11,15$, e.g.]) or because high shear rates are undesirable (for example, dissolved protein characterised by a fragile three dimensional molecular structure [13]).

Boyland et al. [2] considered the stirring of viscous fluid within an open cylindrical vat by means of cylindrical rods embedded within the fluid. An abundance of theoretical, numerical and experimental evidence has now been adduced to the effect that the efficiency of a stirring protocol is principally a function of the topology of the stirrer motion rather than its energy cost.

\subsection{The 2D pigtail protocol}

Figure 1 illustrates one of the simplest mixing protocols, the pigtail protocol for three stirring rods. Each period of this protocol entails two stirrer interchanges. First, the two left-hand rods are interchanged along a clockwise trajectory (denoted $\sigma_{1}$ ); second, the two right-hand rods are interchanged along an anticlockwise trajectory (denoted $\sigma_{2}^{-1}$ ). Figure 2 shows another three stirrer protocol, the clockwise protocol $\sigma_{1} \sigma_{2}$ corresponding to clockwise interchange of the two left-hand rods followed by clockwise interchange of the two righthand rods. Figure 3 presents a stylised rendition of two periods of the pigtail 

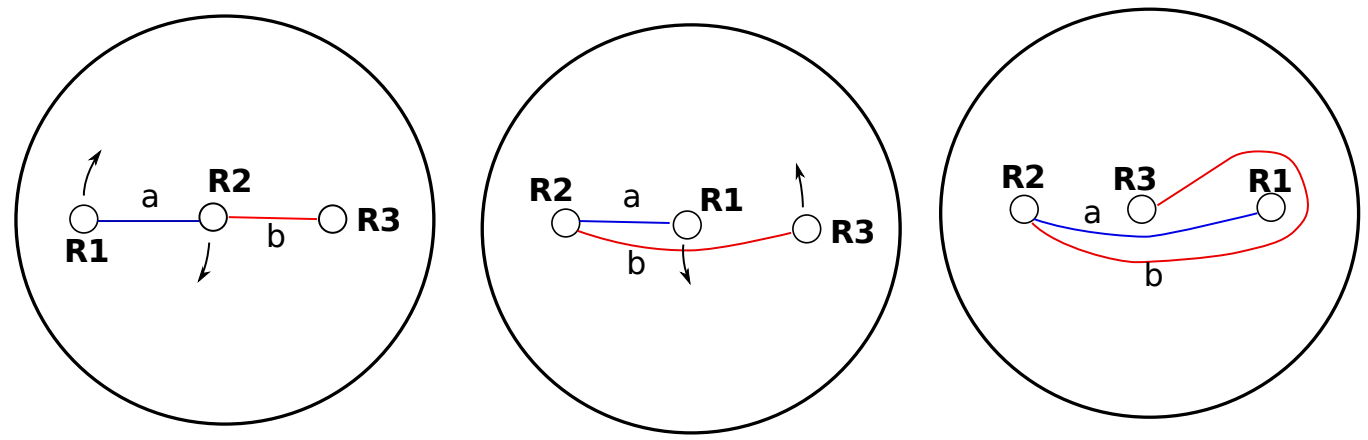

Figure 1: The three stirrer pigtail protocol in a cylindrical vat of fluid. The stirring rods $\left\{R_{1}, R_{2}, R_{3}\right\}$ are indicated by circles. Each period of the protocol comprises two phases: first, the two left-hand rods $R_{1}$ and $R_{2}$ are interchanged along a clockwise trajectory (left to middle figures); second, the new righthand rods $R_{1}$ and $R_{3}$ are interchanged along an anticlockwise trajectory (middle to right). The entropy of this protocol is determined by the action of the flow on filaments connecting $\left(R_{1}, R_{2}\right)$ and $\left(R_{2}, R_{3}\right)$. In particular, the right-hand configuration $(a, b)$ is related to the left-hand configuration $\left(a_{0}, b_{0}\right)$ by the congruence (1) and transition matrix (5), which in turn yield the entropy (6).

protocol (left) and clockwise protocol (right).

Figures 1 and 3 suggest that the pigtail protocol is highly efficient, inducing Fibonacci style layering of filaments. Topologically it is of the form

$$
a \cong a_{0}+b_{0}, \quad b \cong a_{0}+2 b_{0} \quad \text { (up to isotopy) },
$$

being a composition of the $\sigma_{1}$ mapping

$$
\left(\begin{array}{l}
a \\
b
\end{array}\right) \cong \mathbf{T}\left(\sigma_{1}\right)\left(\begin{array}{l}
a_{0} \\
b_{0}
\end{array}\right), \quad \mathbf{T}\left(\sigma_{1}\right)=\left(\begin{array}{ll}
1 & 0 \\
1 & 1
\end{array}\right),
$$

and $\sigma_{2}^{-1}$ mapping:

$$
\left(\begin{array}{l}
a \\
b
\end{array}\right) \cong \mathbf{T}\left(\sigma_{2}^{-1}\right)\left(\begin{array}{l}
a_{0} \\
b_{0}
\end{array}\right), \quad \mathbf{T}\left(\sigma_{2}^{-1}\right)=\left(\begin{array}{ll}
1 & 1 \\
0 & 1
\end{array}\right) .
$$


This protocol induces exponential stretching of nontrivial material loops around pairs of stirrers as a function of time. Furthermore, the efficiency is topological in the sense that it is insensitive to the precise exchange paths of the stirrers (for example, circular versus elliptic or square), stirrer positions (for example, whether collinear and equispaced) or stirrer shape. To be precise, it can be shown that there exists a lower bound $\lambda>1$, called the dilatation, by which certain fluid filaments are stretched from period to period in the large time limit. It is no coincidence that the dilatation coincides with the largest eigenvalue

$$
\lambda=\frac{1}{2}(3+\sqrt{5}) \approx 2.618
$$

of the transition matrix $\mathbf{T}$ defined by (1):

$$
\mathbf{T}\left(\sigma_{1} \sigma_{2}^{-1}\right)=\left(\begin{array}{ll}
1 & 1 \\
1 & 2
\end{array}\right) .
$$

The logarithm of the entropy is referred to as the entropy of the stirring protocol:

$$
\mathrm{h}=\log \lambda=0.962 .
$$

The transition matrices are right multiplicative, satisfying

$$
\mathbf{T}\left(\sigma_{1} \sigma_{2}^{-1}\right)=\mathbf{T}\left(\sigma_{1}\right) \mathbf{T}\left(\sigma_{2}^{-1}\right) .
$$

They act on the nonnegative row-vector

$$
\mathbf{n}=\left(\mathrm{n}_{\mathrm{a}}, \mathrm{n}_{\mathrm{b}}\right)
$$

where $n_{a}$ and $n_{b}$ count the number of $a_{0}$-type and $b_{0}$-type filaments respectively:

$$
\mathbf{n}^{(i+1)}=\mathbf{n}^{(\mathfrak{i})} \mathbf{T}^{(\mathfrak{i})} .
$$

\subsection{The $2 \mathrm{D}$ clockwise protocol}

We now consider the clockwise protocol. It has the same energy cost as the pigtail protocol (at least in the zero inertia limit), differing only in orientation. 
Figure 2 suggests a mapping of the form

$$
\left(\begin{array}{l}
a \\
b
\end{array}\right) \cong \mathbf{T}\left(\sigma_{1} \sigma_{2}\right)\left(\begin{array}{l}
a_{0} \\
b_{0}
\end{array}\right), \quad \mathbf{T}\left(\sigma_{1} \sigma_{2}\right)=\left(\begin{array}{ll}
1 & 1 \\
1 & 0
\end{array}\right)
$$

which in turn suggests an entropy precisely half that of the pigtail protocol:

$$
\lambda=\frac{1}{2}(1+\sqrt{5}) \approx 1.618, \quad \mathrm{~h} \approx 0.481 \quad \text { (incorrect) } .
$$

However, this entropy is incorrect: the clockwise protocol is not guaranteed to produce any exponential stretching - equivalently, to possess positive entropybased only on the relative motion of the stirrers. Since the nonnegative transition matrices fail to distinguish between clockwise and anticlockwise motion, that is,

$$
\mathbf{T}\left(\sigma_{1}^{-1}\right)=\mathbf{T}\left(\sigma_{1}\right) \text { and } \quad \mathbf{T}\left(\sigma_{2}\right)=\mathbf{T}\left(\sigma_{2}^{-1}\right),
$$

they are not always valid under multiplication:

$$
\mathbf{T}\left(\sigma_{1} \sigma_{2}^{-1}\right)=\mathbf{T}\left(\sigma_{1}\right) \mathbf{T}\left(\sigma_{2}^{-1}\right) \quad \text { but } \quad \mathbf{T}\left(\sigma_{1} \sigma_{2}\right) \neq \mathbf{T}\left(\sigma_{1}\right) \mathbf{T}\left(\sigma_{2}\right) .
$$

In particular, they fail to capture the 'snap-back' phenomenon of the bfilament during the $\sigma_{2}$ operation (Figure 2, middle to right), and of both filaments during the third half-period (Figure 3, left). Higher order transition matrices confirm that the clockwise protocol induces circulatory motion, and return a trivial lower bound of $e=0$ on the entropy:

$$
\mathbf{T}\left(\sigma_{1} \sigma_{2} \sigma_{1}\right)=\left(\begin{array}{ll}
0 & 1 \\
1 & 0
\end{array}\right), \quad \mathbf{T}\left(\left(\sigma_{1} \sigma_{2}\right)^{3}\right)=\mathbf{I}
$$

Fortunately, rigorous algorithms are available for entropy determination. However, before outlining these I clarify the relationship between maps, braids and stirring protocols. 

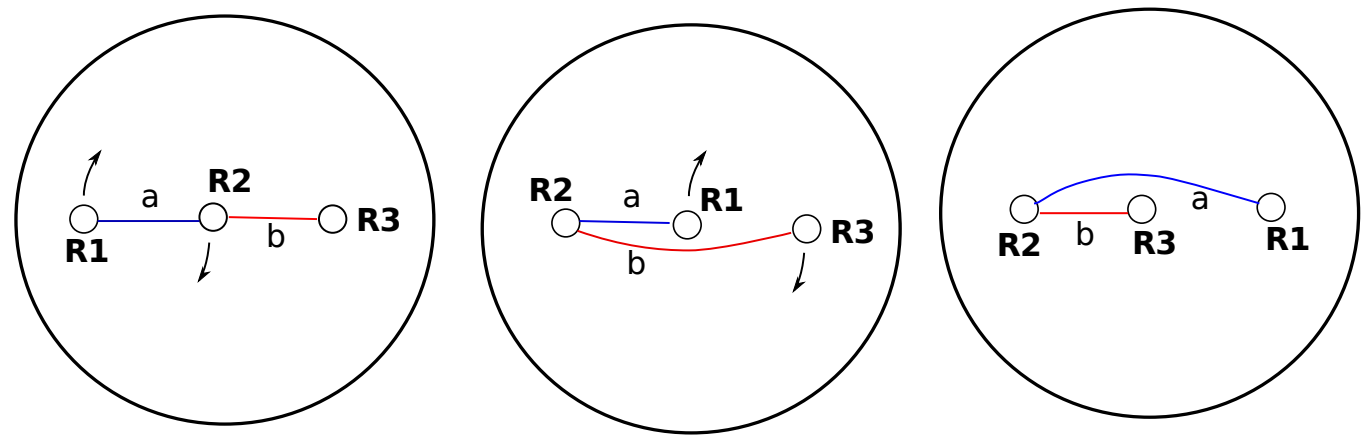

Figure 2: The three stirrer clockwise protocol in a cylindrical vat of fluid. The stirring rods $\left\{R_{1}, R_{2}, R_{3}\right\}$ are indicated by circles. Each period of the protocol comprises two phases: first, the two left-hand rods $R_{1}$ and $R_{2}$ are interchanged along a clockwise trajectory; second, the new right-hand rods $R_{2}$ and $R_{3}$ are interchanged clockwise.

\subsection{Braids}

It is helpful to visualise an n-stirrer protocol as an n-strand braid-see, for example, Figure 6 by Boyland et al. [2] and Figures 1 and 2 by Thiffeault \& Finn [12]. Such a braid is of the form $\left\{\left(x_{j}, y_{j}, t\right)\right\}_{j=1}^{n}$, and corresponds to the parameterised stirrer trajectories $\left\{\left(x_{i}(t), y_{i}(t)\right)\right\}$ plotted against a vertical time axis. Informally, the braid representation explains why the pigtail protocol is topologically complex ('tangled') whereas the clockwise protocol is trivial ('not tangled'). In braiding theory, parametrisation is not important: in a side view of the strands, only the pairwise crossings are deemed significant. Thus, an arbitrary braid can be fully represented by an ordered sequence (for example, $\sigma_{1} \sigma_{2}^{-1}$ ) of strand interchanges as viewed from above. Braids may be further simplified via the equivalence ('presentation') rules

$$
\sigma_{j} \sigma_{j+1} \sigma_{j} \equiv \sigma_{j+1} \sigma_{j} \sigma_{j+1} \quad \text { for } \quad 1 \leqslant j<n-1
$$

and

$$
\sigma_{i} \sigma_{j} \equiv \sigma_{j} \sigma_{i} \quad \text { for } \quad|i-j|>1 .
$$


Pigtail protocol
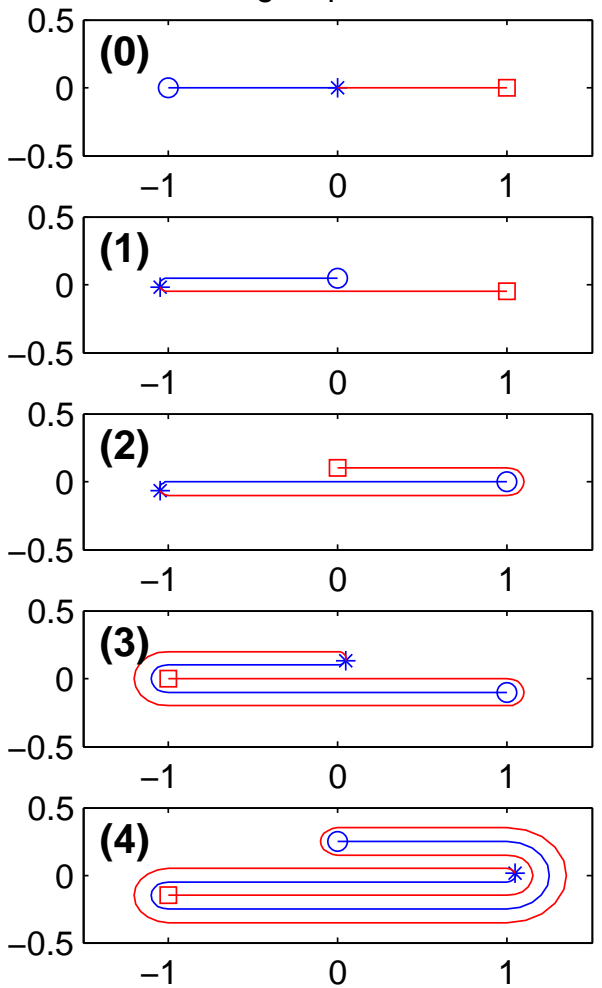
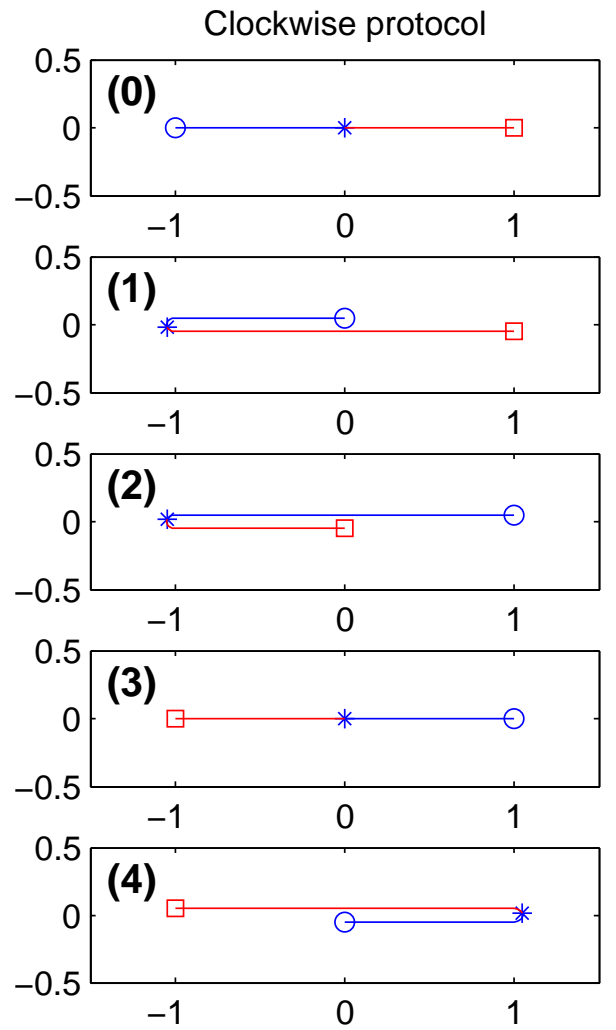

Figure 3: Left: Two periods of the pigtail protocol (Figure 1). Right: Stylised representation of two periods of the clockwise protocol shown in Figure 2.

The braid representation is also useful for rigorous analysis. According to Thurston-Nielsen theory $[2,12]$, there is a one-to-one correspondence between non-equivalent braids and isotopically distinct two dimensional maps. An example of such a map is the inertialess flow field generated by one period of a stirring protocol. Strictly speaking, entropy is a property only of maps (the formal definition being beyond the scope of this article); however, ThurstonNielsen theory justifies the extension of entropy to braids. Thus, the search 
for stirring protocols can be represented conceptually in the form

$$
h_{\text {flow }} \geqslant h_{\text {braid }} \equiv h_{\text {canonical map }} \text {. }
$$

In general, it is a highly nontrivial task to predict a lower bound on the entropy of a flow based solely on the topology of the stirrer motion. However, given a braid representation of the flow one can appeal to braid theory to derive a lower bound (12) on the flow entropy. For general braids, nonnegative transition matrices are clearly inadequate for analysis. Some progress has been made via abstract matrix representations of braids. However, more general algorithms involve a partial return to physical reasoning. The train track algorithm of Bestvina \& Handel [1] is complex, but also appealing inasmuch as it generates an invariant ('train track') representation of the 'shape' of the flow. The filament based algorithm of Moussafir [8], valid for an $\mathrm{n}$-strand braid in a nonperiodic domain, is based on a lamination comprising (one or more) closed loops, such that each loop is wrapped tightly around some (but not all) of the $\mathrm{n}$ stirrers. The status of this lamination after any given braid sequence (periodic or otherwise) is unambiguously represented by an integer valued array of approximate length $2 n$ describing both the thickness and orientation of the filament segments. Moussafir's evolution equations $^{1}$ are thus robust to 'snap-back' or 'unwinding' phenomena. These (slightly tedious) equations are not matrix based, and thus do not yield direct entropy estimates; on the other hand, they are easy to program and converge rapidly to the true braid entropy. The Moussafir algorithm was subsequently extended by Finn \& Thiffeault [4] to the case of $\boldsymbol{n}$ stirrers in a doubly periodic domain (equivalently, on the surface of a torus).

\footnotetext{
${ }^{1}$ Moussafir's terminology [8] is unconventional in several respects: (1) His sign function $\mathrm{a}^{-}$is nonpositive. (2) A clockwise interchange is denoted by $\sigma_{j}^{-1}$, and an anticlockwise one by $\sigma_{j}$. (3) Since the first and last stirrers are stationary, a nontrivial braid requires $n \geqslant 5$ stirrers.
} 


\subsection{Ghost rods}

The robust and efficient stirring of Boyland et al. [2] requires at least three moving rods, since it can be shown that any periodic motion of one or two stirrers in a bounded domain is topologically trivial. However, it was quickly realised that nontrivial flow topology may be induced with fewer than three stirrers. A good example is the figure-of-eight single-stirrer protocol of Gouillart et al. [6], although this may be sensitive to changes in flow parameters. This nontrivial flow is because any periodic point in the flow effectively constitutes a 'virtual stirrer' or 'ghost rod', and thus may be included in any topological analysis of the stirring protocol. For example, a mixing protocol for two dimensional lid driven cavity flow may possess positive entropy even without corresponding to any obvious braid sequence. Moreover, Stremler \& Chen [10] suggested that a modified two dimensional cavity flow, featuring vortical motion with three or more well-defined ghost rods, could be used to obtain effective mixing. Chen \& Stremler [3] further suggested that this vortical lid driven flow could provide efficient lateral mixing for a pressure driven, continuous flow mixer. Though hypothetical at this stage, this continuous flow mixer shows promising potential and bears a strong qualitative resemblance to the realistic micromixer of Kang et al. [7].

\subsection{The next frontier: from $2 \mathrm{D}$ to $3 \mathrm{D}$}

All of the above stirring protocols are essentially two dimensional in nature, since the underlying topological theory (Thurston-Nielsen theory) applies to two dimensional maps. However, guided by these studies we tentatively propose one possible approach to achieving efficient mixing in three dimensions. Section 2 begins by proposing an analogue of braiding in three dimensions. Section 3.1, following the methodology of Stremler and Chen [10, 3], proposes a hypothetical batch mixer capable of executing these three dimensional 'braiding' protocols. The efficiency of each protocol is assessed using surface tracking (Section 3.2) and compared with theoretical predictions (Section 4). 


\section{A theory of $3 \mathrm{D}$ mixing}

\subsection{Advection and topology}

The entropy of a two dimensional flow is defined as the asymptotic minimum growth rate of the length of an arbitrary one dimensional filament advected by the flow. Such a filament may, for example, represent the interface between two chemical species to be mixed. We would like to stretch and fold this filament as rapidly and uniformly as possible to create thin alternating striations of the chemical, thereby accelerating homogenisation by molecular diffusion.

Analogously, in a three dimensional flow we could consider an area of an interfacial surface separating two chemical species. In this situation our aim is to maximise the rate at which this interfacial area is stretched and folded. Accordingly, we use the (asymptotic exponential) area growth rate as a proxy for the entropy of the given flow.

In a two dimensional flow, isolated point stirrers and other periodic points of the flow typically constitute topological obstacles to filament advection. If such periodic points execute a nontrivial braiding motion, then they induce a topologically complex deformation of nearby fluid filaments that cannot be smoothly undone without simultaneously moving the stirrers - even with the aid of an arbitrarily complex, possibly unsteady, pressure field. By contrast, isolated periodic points in a three dimensional flow are physical obstacles to surfaces (by determinism) but are not topological obstacles, since their relative motion is insufficient to yield a lower bound on the stretching of surfaces. Consequently, it is necessary to consider other periodic structures, such as periodic curves (for example, manifolds associated with periodic orbits) which may be used to guarantee stretching of surfaces.

The question now arises of how to induce periodic lines within a three dimensional flow. As a first step toward this goal, we extend two dimensional braiding concepts to three dimensional domains by executing the individual two dimensional braid generators in two orthogonal planes of motion. This 
Table 1: The braided stirring protocols studied in this work.

\begin{tabular}{llll}
\hline Name & Label & Braid sequence & Period \\
\hline 2D pigtail & pig2D & $\sigma_{1} \sigma_{2}^{-1}$ & $\mathrm{~T}=2$ \\
3D pigtail (phased) & pig3D & $\sigma_{1} \sigma_{2}^{-1} \Sigma_{1} \Sigma_{2}^{-1}$ & $\mathrm{~T}=4$ \\
3D pigtail (interleaved) & piglL & $\sigma_{1} \Sigma_{1} \sigma_{2}^{-1} \Sigma_{2}^{-1}$ & $\mathrm{~T}=4$ \\
\hline 2D clockwise & clock2D & $\sigma_{1} \sigma_{2}$ & $\mathrm{~T}=2$ \\
3D clockwise (phased) & clock3D & $\sigma_{1} \sigma_{2} \Sigma_{1} \Sigma_{2}$ & $\mathrm{~T}=4$ \\
3D clockwise (interleaved) & clockIL & $\sigma_{1} \Sigma_{1} \sigma_{2} \Sigma_{2}$ & $\mathrm{~T}=4$ \\
\hline
\end{tabular}

concept is illustrated in Figure 4 for a cubic domain with $m=3$ 'line stirrers' $\left\{R_{1}, R_{2}, R_{3}\right\}$ in the $(x, y)$ plane, together with $n=3$ line stirrers $\left\{S_{1}, S_{2}, S_{3}\right\}$ in the $(z, y)$ plane. The $\sigma_{j}(j=1,2)$ operations correspond to clockwise interchange of the $j$ th and $(j+1)$ th stirrers in the $(x, y)$ plane (viewed in the direction of decreasing $z$ ). We also define $\Sigma_{k}$ operations as the clockwise interchange in the $(z, y)$ plane of the kth and $(k+1)$ th stirrers (viewed in the direction of increasing $x$ ). For example, the $\Sigma_{1}$ generator corresponds in Figure 4 to the clockwise interchange of $S_{1}$ and $S_{2}$, while the $\Sigma_{2}^{-1}$ generator corresponds to anticlockwise interchange of $S_{2}$ and $S_{3}$. By analogy with Thiffeault \& Finn [12], these six line stirrers are considered to form a grid of 'ghost rods', on the understanding that rods $\left\{R_{1}, R_{2}, R_{3}\right\}$ are distorted by motion of $\left\{\mathrm{S}_{1}, \mathrm{~S}_{2}, \mathrm{~S}_{3}\right\}$ and vice versa.

We now define a three dimensional braid to be a finite, repeatable sequence of the generators

$$
\left\{\sigma_{j}^{ \pm 1}, \Sigma_{k}^{ \pm 1}: 1 \leqslant j<m, 1 \leqslant k<n\right\}
$$

and a three dimensional mixing protocol as an implementation of (13) by means of (real or virtual) rods within a body of fluid. The six 3D protocols considered in this article are listed in Table 1 . They comprise three variants of each of these two protocols:

1. quasi- $2 D$, comprising only $\sigma$ operations; 


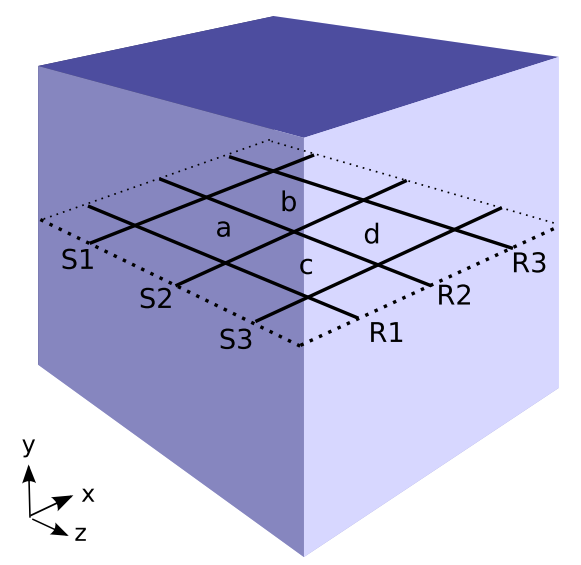

Figure 4: The four surface elements $\{a, b, c, d\}$ defined by a configuration of $m=3$ ghost rods $\left\{R_{1}, R_{2}, R_{3}\right\}$ restricted to move in the $(x, y)$ plane and $n=3$ rods $\left\{S_{1}, S_{2}, S_{3}\right\}$ moving in the $(z, y)$ plane. The general case of $m+n$ stirrers yields a total of $(m-1) \times(n-1)$ area elements.

2. phased or weakly three dimensional, comprising alternating sequences of $\sigma$ and $\Sigma$ operations; and

3. interleaved or strongly three dimensional, comprising alternating sequences of single $\sigma$ and $\Sigma$ operations.

\subsection{A theory of $3 \mathrm{D}$ braids}

Our principal goal is to estimate the dilatation $\lambda$ and entropy $h$ of each protocol listed in Table 1. The formal definition of entropy of a 3D map is highly nontrivial and well beyond the scope of this article. Instead, in an abuse of notation, we define the 'entropy' informally as the exponential growth rate in surface area of a 'typical' surface element advected by the corresponding three dimensional flow.

The $m+n=6$ ghost rods shown in Figure 4 define a set of $(m-1)(n-1)$ 

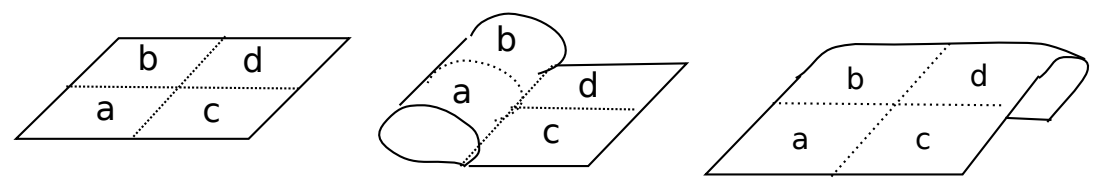

Figure 5: Left: Schematic representation of the surface elements $\{a, b, c, d\}$ from Figure 4 for $3 \times 3$ braids. Middle: Schematic representation of the $\Sigma_{1}$ generator. Right: Schematic representation of the $\sigma_{2}$ generator.

area elements, here labelled $\{a, b, c, d\}$. Figure 5 illustrates schematically the response of these initial elements (left) to the $\Sigma_{1}$ generator (middle) and $\sigma_{2}$ generator (right). The $\Sigma_{1}$ transformation is of isotopic form

$$
\left(\begin{array}{l}
a \\
b \\
c \\
d
\end{array}\right) \cong\left(\begin{array}{llll}
1 & 0 & 0 & 0 \\
0 & 1 & 0 & 0 \\
1 & 0 & 1 & 0 \\
0 & 1 & 0 & 1
\end{array}\right)\left(\begin{array}{l}
a_{0} \\
b_{0} \\
c_{0} \\
d_{0}
\end{array}\right)
$$

The $4 \times 4$ matrix in (14) is of Kronecker product form

$$
\mathbf{S}=\mathbf{T}_{\Sigma} \otimes \mathbf{T}_{\sigma}
$$

with $\mathbf{T}_{\sigma}$ as the $\mathbf{m}$-stirrer identity matrix and $\mathbf{T}_{\Sigma}$ as the $\mathbf{n}$-stirrer transition matrix for the $\sigma_{1}$ operation:

$$
\mathbf{T}_{\Sigma}=\mathbf{T}\left(\sigma_{1}\right)=\left(\begin{array}{ll}
1 & 0 \\
1 & 1
\end{array}\right), \quad \mathbf{T}_{\sigma}=\mathbf{T}\left(\mathrm{id}_{\mathrm{m}}\right)=\left(\begin{array}{ll}
1 & 0 \\
0 & 1
\end{array}\right) .
$$

The $\sigma_{2}$ transformation takes the same form as (14)-(15) with

$$
\mathbf{T}_{\Sigma}=\mathbf{T}\left(\mathrm{id}_{\mathfrak{n}}\right)=\left(\begin{array}{ll}
1 & 0 \\
0 & 1
\end{array}\right), \quad \mathbf{T}_{\sigma}=\mathbf{T}\left(\sigma_{2}\right)=\left(\begin{array}{ll}
1 & 1 \\
0 & 1
\end{array}\right) .
$$

The transition matrix $\mathbf{S}$ is right multiplicative, that is,

$$
\mathbf{n}^{(i+1)}=\mathbf{n}^{(i)} \mathbf{S}^{(i)},
$$


on the filament thickness row-vector

$$
\mathbf{n}=\left(\mathrm{n}_{\mathrm{a}}, \mathrm{n}_{\mathrm{b}}, \mathrm{n}_{\mathrm{c}}, \mathrm{n}_{\mathrm{d}}\right) .
$$

We conjecture that some 'simple' 3D braids are of the separable form (15), with dilatation $\lambda$ corresponding to the dominant eigenvalue of the transition matrix $\mathbf{S}$. (The uniqueness of dilatation $\lambda$ is guaranteed by positivity and the Perron-Frobenius Theorem.) Kronecker product properties then imply that the full-period entropy is simply the sum of the entropies in each plane:

$$
h=h_{\sigma}+h_{\Sigma} \quad \text { (conjectured separable 3D braids). }
$$

The pig3D protocol appears to be of this type, implying an entropy of

$$
\mathrm{h}=2 \times 0.9624=1.925 .
$$

This in turn implies that the pig2D and pig3D protocols are equally efficient in energy terms, as measured by entropy per generator.

We now consider the clock3D protocol. One might expect it to be topologically trivial, like its $2 \mathrm{D}$ counterpart:

$$
\mathrm{h}_{\sigma}=\mathrm{h}_{\Sigma}=0 \Rightarrow \mathrm{h}=0 .
$$

However, recall that the clock2D protocol exhibits cancellation or 'snapback' both within and between mixing periods. We conjecture that its 3D counterpart exhibits only within-period cancellation, owing to alternation of the braiding plane. More precisely, we conjecture with reference to Figure 6 that the clock3D protocol is separable and nontrivial, of the form (14)-(15) with

$$
\mathbf{T}_{\Sigma}=\mathbf{T}_{\sigma}=\mathbf{T}\left(\sigma_{1} \sigma_{2}\right)=\left(\begin{array}{ll}
1 & 1 \\
1 & 0
\end{array}\right), \quad \mathbf{S}=\left(\begin{array}{llll}
1 & 1 & 1 & 1 \\
1 & 0 & 1 & 0 \\
1 & 1 & 0 & 0 \\
1 & 0 & 0 & 0
\end{array}\right)
$$


The separability conjecture then yields

$$
h=2 \times \log \left[\frac{1}{2}(1+\sqrt{5})\right]=\log \left[\frac{1}{2}(3+\sqrt{5})\right]=0.962,
$$

indicating that the clock3D protocol has the same entropy as the pig2D protocol and thus is half as efficient, in energy terms, as either the pig2D or the pig3D protocol. In addition, an upper bound on the braid entropy is suggested by the max-norm of the transition matrices in (22), corresponding to the maximum layer thickness in Figure 6:

$$
\lambda_{\sigma}=\lambda_{\Sigma} \leqslant 2, \quad \lambda \leqslant 4, \quad h \leqslant \log 4=1.386 .
$$

Finally, it appears intuitively reasonable to expect the interleaved braids pigIL and clockIL to possess the same entropies as their phased counterparts pig3D and clock3D respectively. We test this idea by appealing once more to a folding argument. In particular, we conjecture that the phased braids can be represented as the product of two $\mathbf{S}$-matrices, each of which is square with dimension $(m-1)(n-1)$ :

$$
\mathbf{S}\left(\sigma_{1} \sigma_{2}^{ \pm 1} \Sigma_{1} \Sigma_{2}^{ \pm 1}\right)=\mathbf{S}\left(\sigma_{1} \Sigma_{2}^{ \pm 1}\right) \mathbf{S}\left(\Sigma_{1} \sigma_{2}^{ \pm 1}\right) .
$$

Conversely, the (period-4) interleaved braids each require four S-matrices:

$$
\mathbf{S}\left(\sigma_{1} \sigma_{2}^{ \pm 1} \Sigma_{1} \Sigma_{2}^{ \pm 1}\right)=\mathbf{S}\left(\sigma_{1}\right) \mathbf{S}\left(\Sigma_{1}\right) \mathbf{S}\left(\sigma_{2}^{ \pm 1}\right) \mathbf{S}\left(\Sigma_{2}^{ \pm 1}\right) .
$$

It then follows from elementary properties of Kronecker products that

$$
\mathbf{S}\left(\sigma_{1} \sigma_{2}^{ \pm 1} \Sigma_{1} \Sigma_{2}^{ \pm 1}\right)=\mathbf{T}\left(\Sigma_{1} \Sigma_{2}^{ \pm 1}\right) \otimes \mathbf{T}\left(\sigma_{1} \sigma_{2}^{ \pm 1}\right)
$$

for the phased braids, and

$$
\mathbf{S}\left(\sigma_{1} \Sigma_{1} \sigma_{2}^{ \pm 1} \Sigma_{2}^{ \pm 1}\right)=\mathbf{T}\left(\Sigma_{1}\right) \mathbf{T}\left(\Sigma_{2}^{ \pm 1}\right) \otimes \mathbf{T}\left(\sigma_{1}\right) \mathbf{T}\left(\sigma_{2}^{ \pm 1}\right),
$$

for the interleaved braids, where each $\mathbf{T}_{\Sigma}$ matrix is of size $(n-1)$ and each $\mathbf{T}_{\sigma}$ matrix is of size $(m-1)$. 
Initial filament

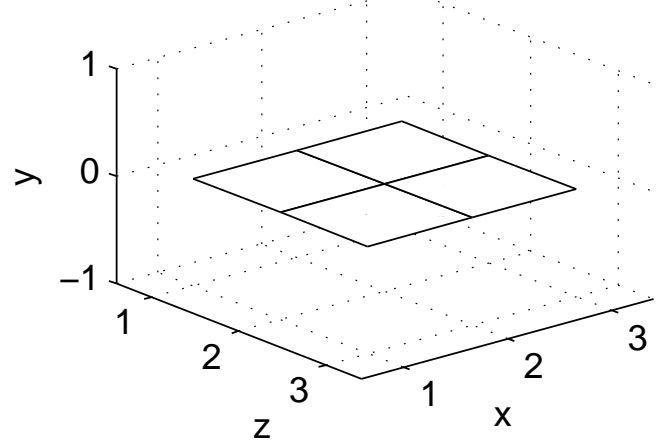

Full-period $\left(\sigma_{1} \sigma_{2} \Sigma_{1} \Sigma_{2}\right)$

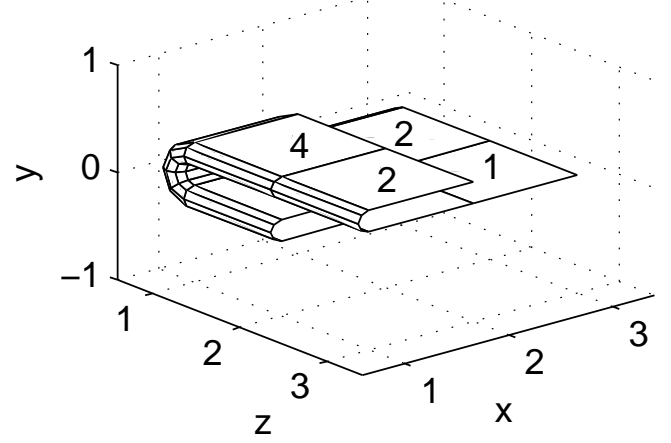

Half-period $\left(\sigma_{1} \sigma_{2}\right)$

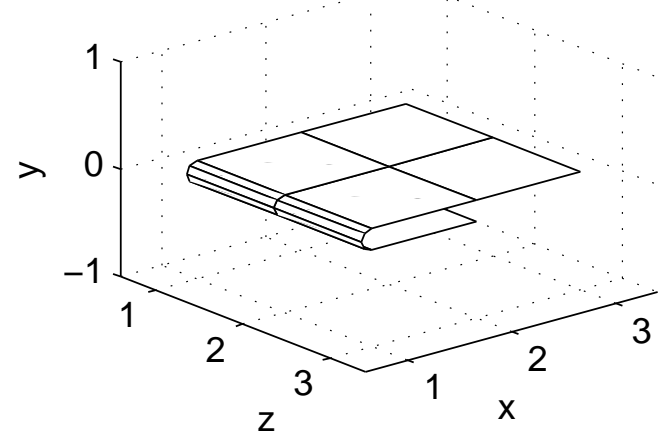

Full-period [viewed from below]

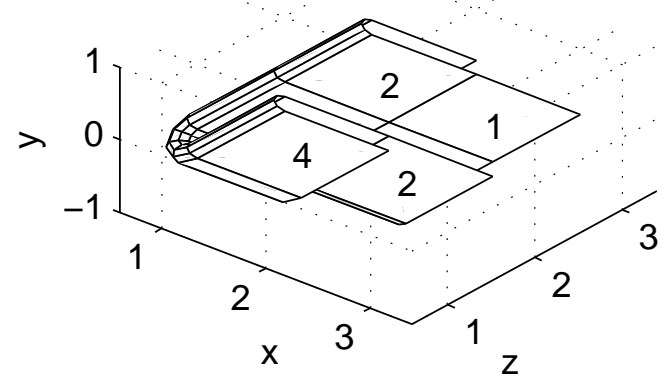

Figure 6: Conjectured behaviour of the 3D phased clockwise braid (clock3D, periodic sequence $\left.\sigma_{1} \sigma_{2} \Sigma_{1} \Sigma_{2}\right)$. The final configuration comprises four area elements of type $\mathrm{a}_{0}$, two of $\mathrm{b}_{0}$, two of $\mathrm{c}_{0}$ and one of $\mathrm{d}_{0}$ (lower-left and lowerright figures, with filament thicknesses as indicated). This is consistent with the Kronecker matrix representation (15), (18), (19) and (22). 
Equations (15) and (27) are equivalent, yielding the same entropy estimates (21) and (23) for the pig3D and clock3D protocol respectively. Similarly, (15) and (28) are equivalent for the piglL protocol, indicating equal entropies for the pig3D and pigIL protocols. On the other hand, (15) and (28) are not equivalent for the clockIL protocol. More precisely, (28) predicts that the clockIL protocol has the same entropy as the pig3D and pigIL protocols and twice the entropy of the clock3D protocol. This implies that the four protocols pig2D, pig3D, pigIL and clockIL are equally efficient in energy terms.

\subsection{Practical issues: from braids to protocols}

In Section 2 we outlined a theory of 3D braiding for a planar array of $m+n$ stirrers. We proceeded to propose 3D mixing protocols and corresponding entropy estimates for the case $m=n=3$. We must now propose plausible flows and practical stirring mechanisms for implementation of these protocols.

These practical difficulties are admittedly formidable. For example, are the stirrers $\left\{R_{1}, R_{2}, R_{3}\right\}$ and $\left\{S_{1}, S_{2}, S_{3}\right\}$ in Figure 4 to be interpreted as physical rods - or are they strictly virtual, denoting 'ghost rods' identifiable within a 3D flow field?

One could naively imagine a physical implementation of Figure 4 using rigid but retractable rods capable of insertion into, and withdrawal from, the fluid as required. For example, the phased protocols (pig3D or clock3D) could be implemented using three rods, which would presumably be inserted twice and removed twice during each period of mixing. The process of insertion and withdrawal would itself modify the flow field, especially in the Stokes flow limit of high viscosity and negligible inertia. However, more troublesome is the practical problem of mounting the stirrers so that they are free to move both vertically and horizontally. For example, could they be arranged to slide along rigid lateral walls of the mixing chamber? Even if this were possible, the resulting flow field would be theoretically singular - not an uncommon phenomenon in real world modelling, but potentially hazardous 
from a numerical perspective.

We regard the stirrers as virtual, and Figure 4 as idealising either an improved continuous flow micromixer or a lid driven batch mixer. In either case, the objective is to induce alternating asymmetric vortical flows qualitatively similar to the 3D braids specified in Table 1. In the batch mixer case, the driving mechanism comprises one or more sliding cavity walls or lids - possibly sectioned lids, as proposed by Stremler \& Chen [10] —inducing a viscous flow field subject to no-slip conditions on all boundaries. In the continuous flow case, at least two plausible mechanisms are available: physical and electrostatic. The physical mechanism, acting through a no-slip boundary condition, comprises microscale corrugations or other 3D micro-channel structures, such as those studied by Kang [7]. Alternatively, electrostatic forces are used either to drive a continuous flow micromixer or to induce lateral motion within such a flow (Whitesides \& Stroock [15]). Electrostatic forcing acts through nanoscale boundary layers; the resulting flow is effectively macroscopic, corresponding to free slip vortical motion along a rigid boundary.

Whatever the chosen mechanism-lid motion, static corrugations and electrostatic fields - the $(x, y)$ or $(z, y)$ advection of Figure 4 would most likely be applied to the top and/or bottom faces of the cavity, rather than on the indicated side walls. However, the interchange of ghost rods is only approximate at best: in the language of microfluidics, the local turning angle (Whitesides \& Stroock [15]) is a function of position, rather than a uniform 180 degrees as indicated in Figure 4. Similarly, the manifold of periodic points of the flow is curved rather than rod-like. 


\section{Method}

\subsection{A hypothetical batch mixer}

Our ultimate aim is to apply 3D braid theory to the design of practical mixers (either batch based or continuous flow). As a precursor to this computationally challenging task, we now propose a hypothetical batch mixer corresponding as closely as possible to the idealised braiding mixer of Figure 4. In this hypothetical inertialess flow, free-slip boundary conditions apply on the cavity walls in contact with the ghost rods; these correspond to $z= \pm 1$ in the case of $\sigma$ operations and to $x= \pm 1$ for $\Sigma$ operations. Rigid no-slip boundary conditions are enforced on all other cavity walls, including the top and bottom faces of the cavity $(y= \pm 1)$.

We must now define eight hypothetical flows, corresponding to the eight braid generators in (13) and Table 1. Fortunately, all eight can be readily defined with respect to a single 'basic' flow field with an analytic, closed form representation.

The basic flow in our hypothetical batch mixer is plotted in the $(x, y)$ plane in Figure 7 (left). This flow implements the $\sigma_{1}$ operation within the double unit cubic domain $\mathcal{D}=[-1,1]^{3}$; it is expressible by a single streamfunction $\psi$ of the form

$$
\begin{aligned}
(u, v, w) & =\left(\psi_{y},-\psi_{x}, 0\right) \\
\psi\left(x, y, z ; \sigma_{1}\right) & =\beta(x-\alpha)\left(1-x^{2}\right)^{2}\left(1-y^{2}\right)^{2},
\end{aligned}
$$

where the geometric parameter $0<\alpha<1$ and scale factor $\beta>0$ are to be defined shortly. The three starred points in Figure 7 (left) indicate the respective rest positions $\left\{\left(x_{j}, y_{j}\right)=\left(a_{j}, 0\right)\right\}$ of the ghost rods or line stirrers $\left\{R_{1}, R_{2}, R_{3}\right\}$ in Figure 4. In particular, we identify $R_{3}$ with the right-hand stagnation point within the clockwise rotating minor eddy occupying the right-hand sub-domain $\alpha<x<1$. The other two ghost rods $\left\{R_{1}, R_{2}\right\}$ are 

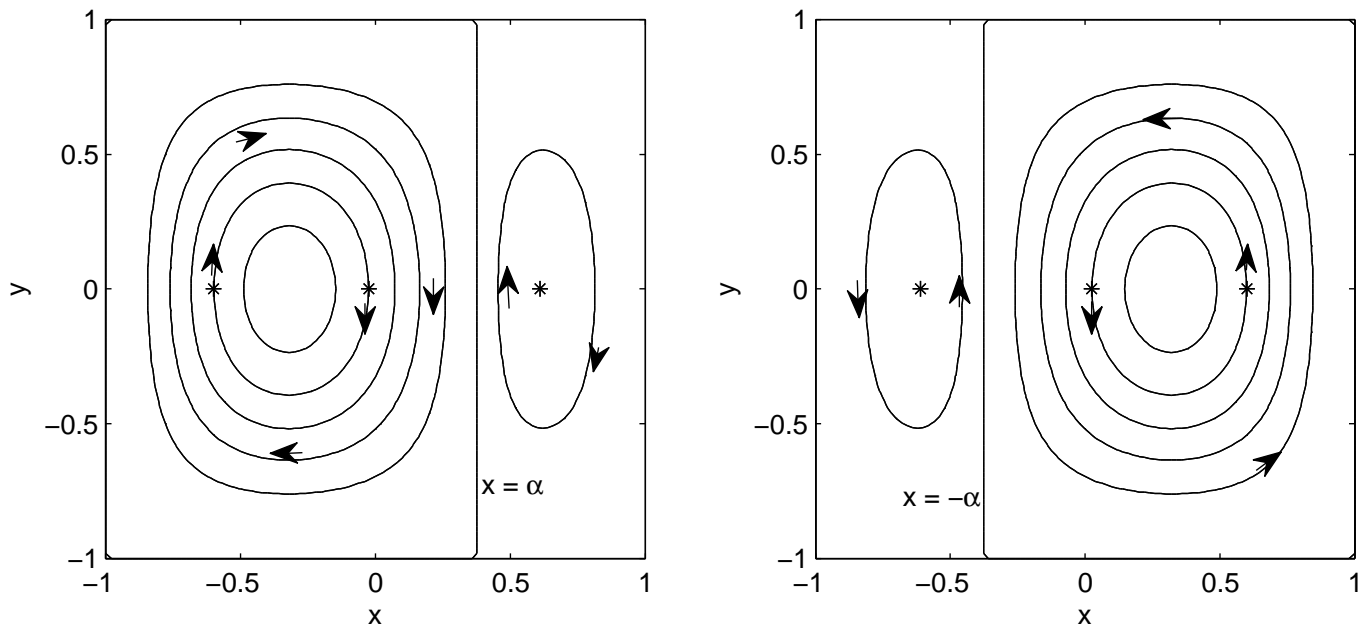

Figure 7: Planar streamlines of the hypothetical three dimensional laminar flow defined in Section 3.1 for implementation of the stirring protocols of Table 1. Left: Streamlines for the $\sigma_{1}$ braid generator in the $(x, y)$ plane, corresponding to the interchange of $\mathrm{R}_{1}$ and $\mathrm{R}_{2}$ in Figure 4; the corresponding flow field is specified by (29). The three starred points denote the rest positions $\left\{\left(a_{j}, 0\right)\right\}$ of the ghost rods $\left\{R_{1}, R_{2}, R_{3}\right\}$ in Figure 4 . In the special case $\alpha=0.3751$ these rods are symmetrical, with $\left(a_{1}, a_{2}, a_{3}\right)=(-a, 0, a)$ for $a=0.6217$. Right: Streamlines for the $\sigma_{2}^{-1}$ streamfunction (31), obtained by reflection of the $\sigma_{1}$ streamfunction in the $y$-axis. Starred points correspond to ghost rods $\left\{R_{1}^{\prime}, R_{2}^{\prime}, R_{3}^{\prime}\right\}$; for $\alpha=0.3751$ they coincide with the ghost rods $\left\{R_{1}, R_{2}, R_{3}\right\}$ at left. The $\Sigma_{2}^{-1}$ generator, corresponding to the interchange of $S_{2}$ and $S_{3}$ in Figure 4, is implemented by mapping the above $\sigma_{2}^{-1}$ flow to the $(z, y)$ plane. 
identified with a streamline of the anticlockwise major eddy occupying the left-hand sub-domain $-1<x<\alpha$.

The other braiding operators are derived from the $\sigma_{1}$ operation as follows. Reversal of the $\sigma_{1}$ flow yields the inverse $\sigma_{1}^{-1}$ operation:

$$
\psi\left(x, y, z ; \sigma_{1}^{ \pm 1}\right)= \pm \beta(x-\alpha)\left(1-x^{2}\right)^{2}\left(1-y^{2}\right)^{2} .
$$

The $\sigma_{2}$ and $\sigma_{2}^{-1}$ operations are obtained by $y$-wise reflection of the $\sigma_{1}^{-1}$ and $\sigma_{1}$ operations respectively:

$$
\psi\left(x, y, z ; \sigma_{2}^{ \pm 1}\right)=\mp \beta(x+\alpha)\left(1-x^{2}\right)^{2}\left(1-y^{2}\right)^{2} .
$$

The $\sigma_{2}^{-1}$ streamfunction is plotted in Figure 7 (right). The three starred points in this figure correspond to ghost rods $\left\{R_{1}^{\prime}, R_{2}^{\prime}, R_{3}^{\prime}\right\}$ with respective rest positions $\left\{\left(x_{j}^{\prime}, y_{j}^{\prime}\right)=\left(a_{j}^{\prime}, 0\right)\right\}$. We wish to ensure that these ghost rods coincide with those of the $\sigma_{1}$ operation, that is, $R_{j}^{\prime}=R_{j}$ and $a_{j}^{\prime}=a_{j}$ for $j=1,2,3$. This property will hold automatically if the $\sigma_{1}$ ghost rods are symmetric, that is, $\left(a_{1}, a_{2}, a_{3}\right)=(-a, 0, a)$ for some constant $\alpha<a<1$. We satisfy this additional condition by numerically solving for $\alpha$ inversely as a function of $a$ in the form

$$
\psi\left(-a(\alpha), 0 ; \sigma_{1}\right)=\psi\left(0,0 ; \sigma_{1}\right) \text { where } a(\alpha)=\operatorname{argmax}_{x} \psi\left(x, 0 ; \sigma_{1}\right),
$$

to yield

$$
\alpha=0.3751, \quad a=0.6217 .
$$

Next, the scaling is set to

$$
\beta=1.3148
$$

so that the duration of each $\sigma_{j}$ operation is precisely one time unit. Finally, the transverse $\Sigma_{k}$ operations follow directly from their $\sigma_{k}$ counterparts via the coordinate interchange $(x, u) \rightarrow(z, w)$. Thus, the overall flow field is piecewise steady; more precisely, it is steady within each generator and varies between generators only in sign and spatial orientation. 
clock3D
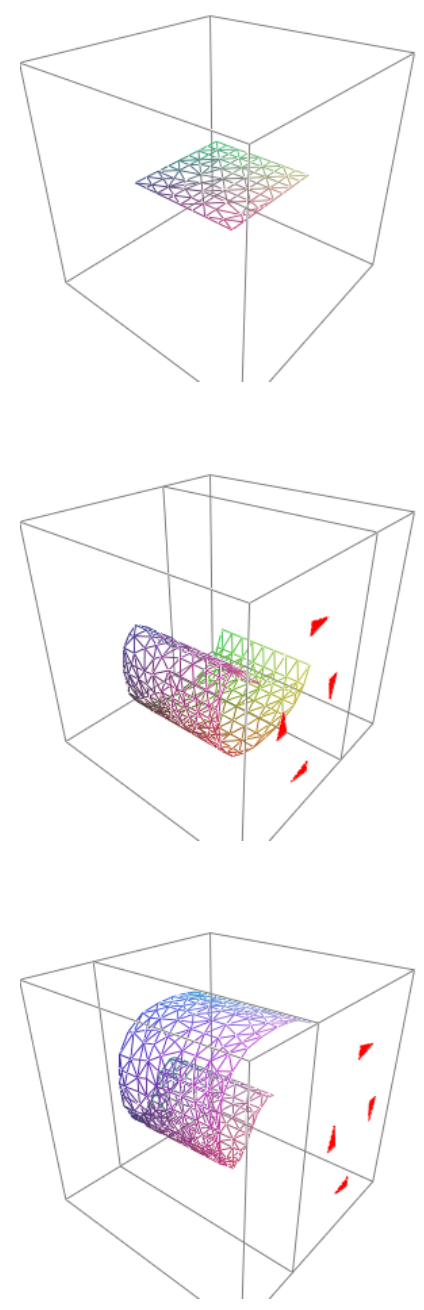

pig3D

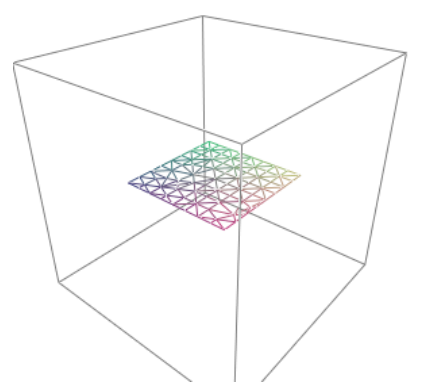

$\mathrm{t}=\mathrm{o}$

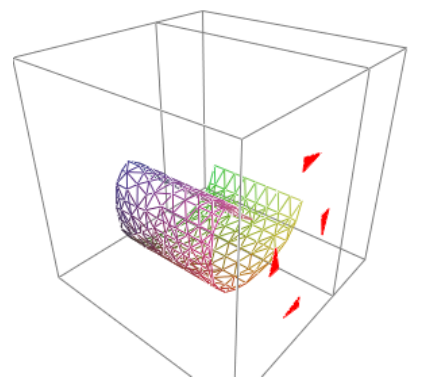

$\mathrm{t}=1$

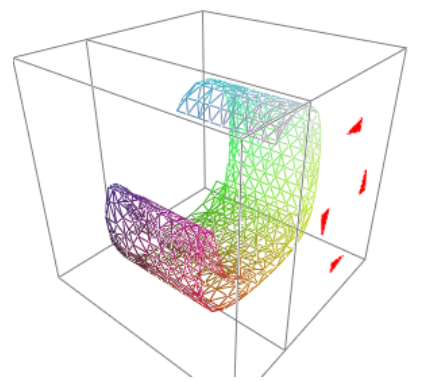

$\mathrm{t}=2$
pigIL
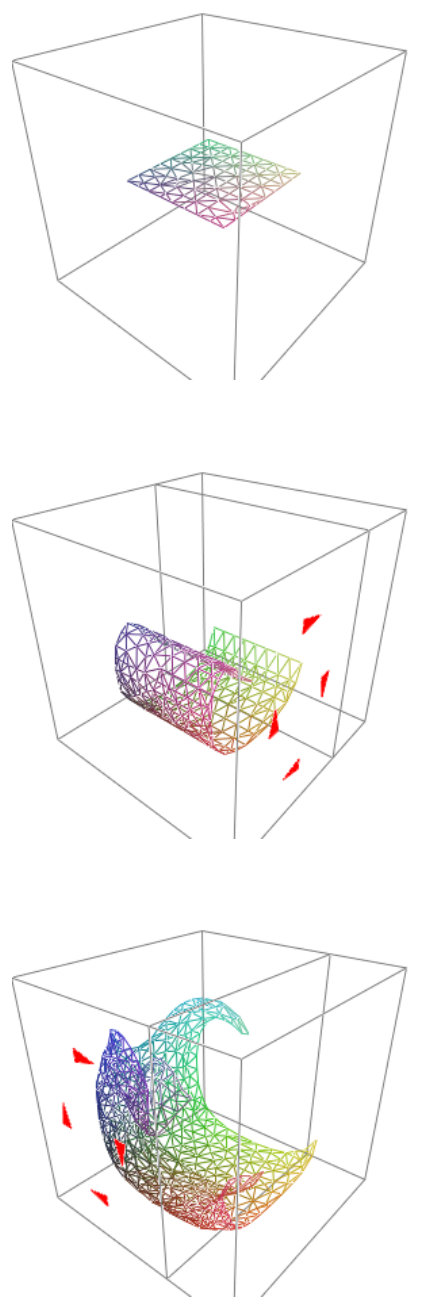

Figure 8: Advection of a reference surface $\mathcal{S}_{0}(t=0)$ by the first half-period $(\mathrm{t}=0,1,2)$ of the clock3D (left), pig3D (middle) and pigIL (right) protocols. Arrowheads indicate the direction of ghost rod interchange within each phase. The initial surface $\mathcal{S}_{0}$ is a centred horizontal square of half-width $s=0.6217$, corresponding to the filament $\{\mathrm{a}, \mathrm{b}, \mathrm{c}, \mathrm{d}\}$ of Figure 4. 
clock3D
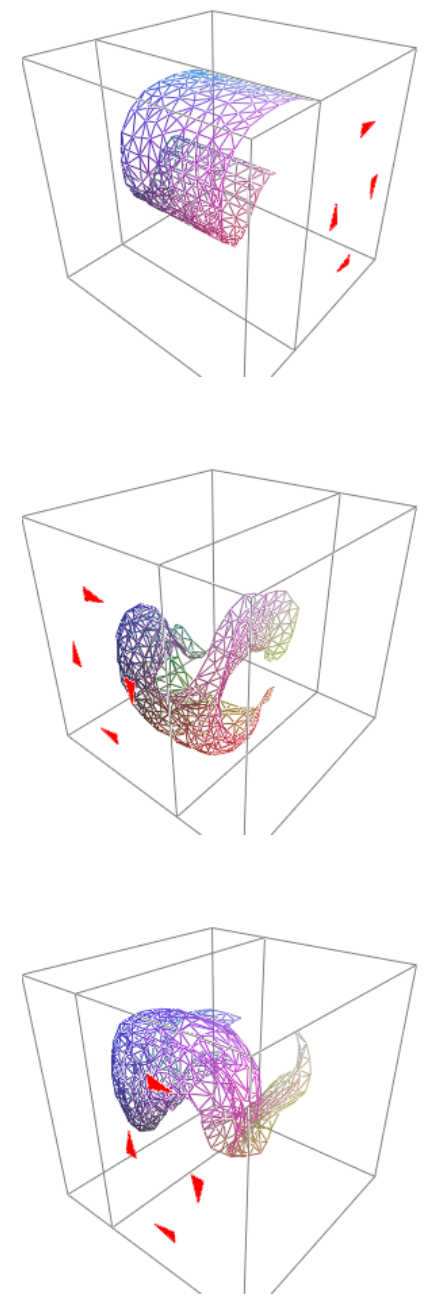

pig3D

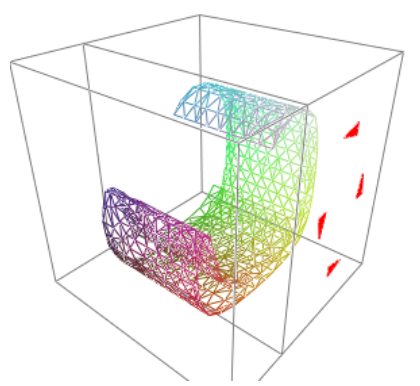

$t=2$

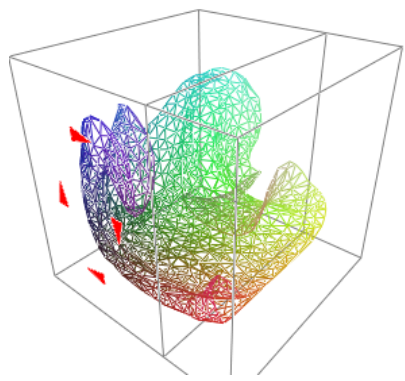

$$
\mathrm{t}=3
$$

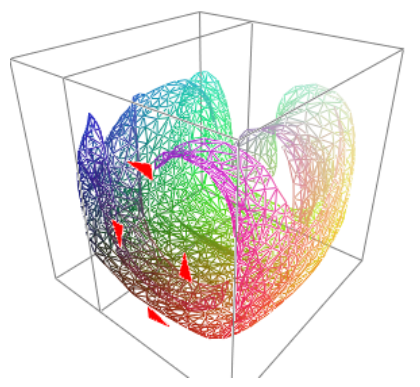

$$
\mathrm{t}=4
$$

pigIL
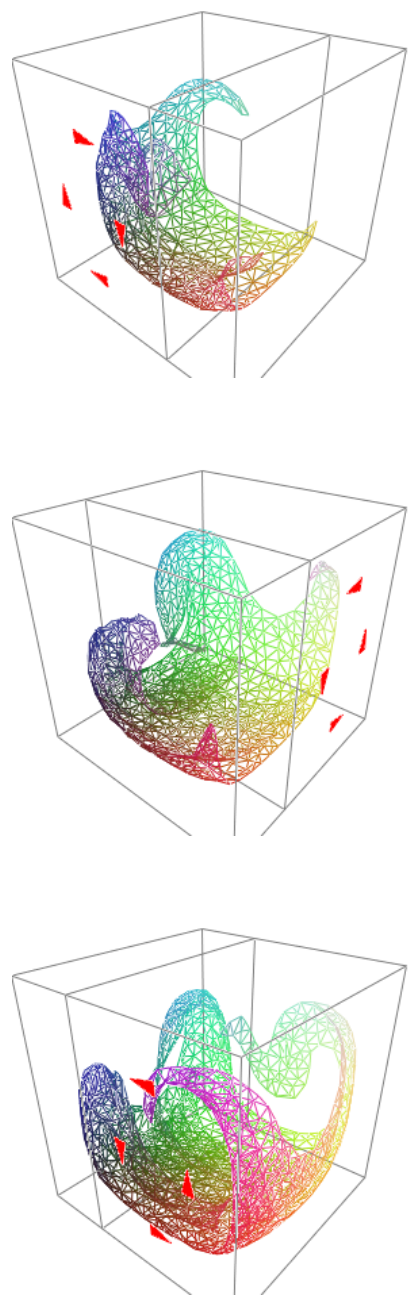

Figure 9: Advection of $\mathcal{S}_{0}$ by the second half-period $(t=2,3,4)$ of the clock3D, pig3D and piglL protocols. 


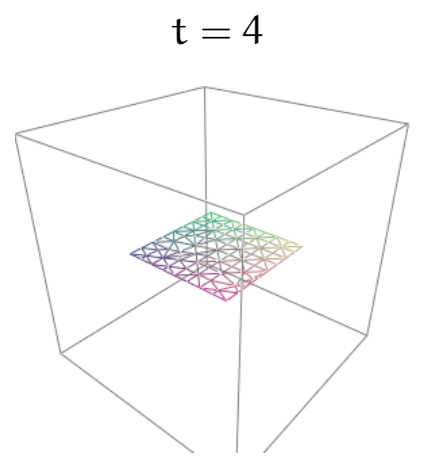

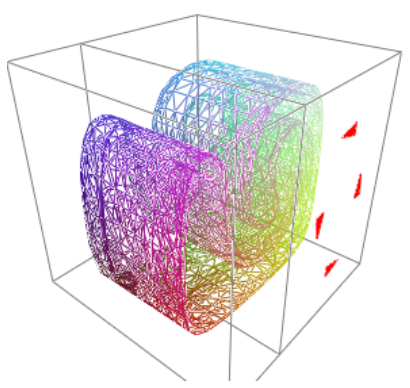

pig2D

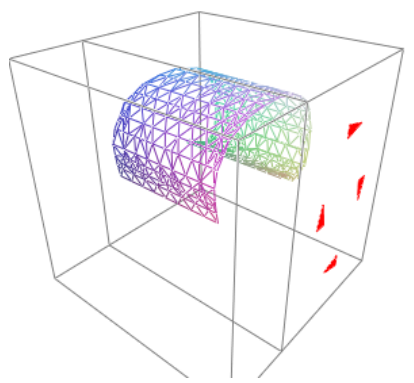

clock2D

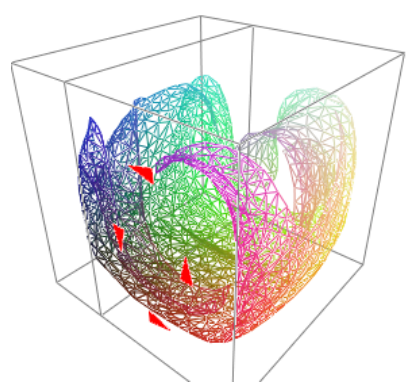

pig3D

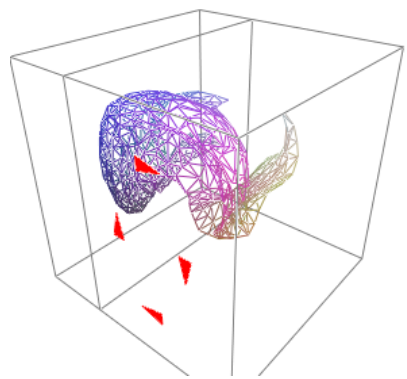

clock3D

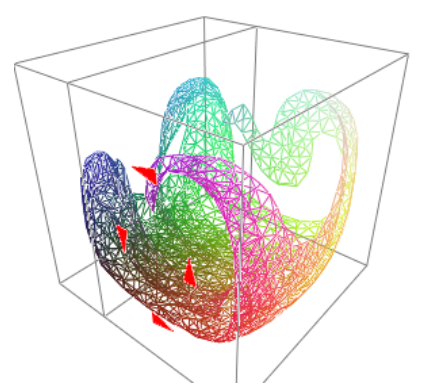

pigIL

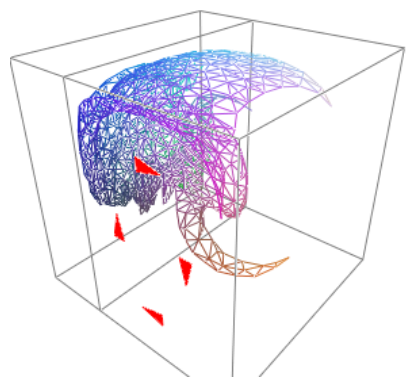

clockIL

Figure 10: Advected surface $\mathcal{S}(t)$ for $t=4$ under each of the six protocols as indicated. 


\subsection{Surface tracking algorithm}

We use the batch mixer of Section 3.1 to study the braided stirring protocols with $\mathrm{m}=3$ line stirrers in the $(x, y)$ plane and $n=3$ line stirrers in the transverse $(z, y)$ plane, as specified in Table 1. Each protocol thus defines a periodic sequence of piecewise steady flows. The efficacy of each stirring protocol is assessed as follows. We choose a reference surface $\mathcal{S}(0) \equiv \mathcal{S}_{0}$ within the interior of the domain. The chosen protocol then transforms $\mathcal{S}(0)$ into a time varying surface $\mathcal{S} \equiv \mathcal{S}(\mathrm{t})$, as shown in Figures 8 to 10 . The relative area $A(t) / A_{0}$ of $\mathcal{S}(t)$ typically exhibits exponential growth as a function of time $t$, thereby furnishing an estimate of the corresponding braid entropy. Each surface $\mathcal{S}(t)$ is represented as an unstructured triangular mesh $\mathcal{N}(t)$ of passively advected particles. Particle trajectories are computed using standard fourth order Runge-Kutta integration. Localised refinement of the stretched mesh is performed using triangle bisection (with optional local re-meshing) as outlined by Galaktionov et al. [5] and Unverdi \& Tryggvason [14]. For a chaotic flow, this refinement protocol ensures exponential growth in the number $\mathrm{N}(\mathrm{t})$ of component particles in $\mathcal{M}(\mathrm{t})$.

Ideally, the empirical entropy rate for any given stirring protocol should be independent of meshing parameters and choice of initial surface $\mathfrak{S}_{0}$. However, in practice finite horizon effects are significant, and the exponential area growth of $\mathcal{S}(t)$ necessitates some trade-off between spatial and temporal resolution. For each simulation, our computing resources impose a practical limit of $\mathcal{O}\left(10^{8}\right)$ for representation of $\mathcal{S}(t)$. Qualitative results were derived using relatively coarse meshes, with $\mathcal{S}_{0}$ varying in size, position and shape (cube, sphere, rectangle). Quantitative results were computed using a fine mesh and spherical initial surface $\mathcal{S}_{0}=\mathcal{B}$ of radius 0.2 , suggestive of a bubble of dye embedded in a fluid filled cavity (Figures 11 and 12). For an initial surface of this size, a mesh $\mathcal{M}(t)$ is considered fine if, for all $t$, the maximum separation distance between adjacent particles is 0.04 , that is, $2 \%$ of the cavity width. 
Table 2: Qualitative ranking (best to worst) of the stirring protocols, based on Figures 8 to 12.

\begin{tabular}{cll}
\hline$=1$ & pig3D & $\sigma_{1} \sigma_{2}^{-1} \Sigma_{1} \Sigma_{2}^{-1}$ \\
$=1$ & pigIL & $\sigma_{1} \Sigma_{1} \sigma_{2}^{-1} \Sigma_{2}^{-1}$ \\
2 & clockIL & $\sigma_{1} \Sigma_{1} \sigma_{2} \Sigma_{2}$ \\
3 & clock3D & $\sigma_{1} \sigma_{2} \Sigma_{1} \Sigma_{2}$ \\
4 & pig2D & $\sigma_{1} \sigma_{2}^{-1}$ \\
5 & clock2D & $\sigma_{1} \sigma_{2}$ \\
\hline
\end{tabular}

Table 3: Tentative quantitative ranking of the stirring protocols (see Figure 13), using empirical entropy rate $\tilde{h} / \mathrm{T}$ as a proxy for energy efficiency.

\begin{tabular}{lll}
\hline 1 & pig2D & $\sigma_{1} \sigma_{2}^{-1}$ \\
2 & pig3D & $\sigma_{1} \sigma_{2}^{-1} \Sigma_{1} \Sigma_{2}^{-1}$ \\
3 & pigIL & $\sigma_{1} \Sigma_{1} \sigma_{2}^{-1} \Sigma_{2}^{-1}$ \\
4 & clockIL & $\sigma_{1} \Sigma_{1} \sigma_{2} \Sigma_{2}$ \\
5 & clock3D & $\sigma_{1} \sigma_{2} \Sigma_{1} \Sigma_{2}$ \\
6 & clock2D & $\sigma_{1} \sigma_{2}$ \\
\hline
\end{tabular}

\section{Results}

Qualitative results for the six mixing protocols are presented in Figures 8 to 12 and summarised in Table 2. The pig3D and pigIL protocols are approximately equivalent except over short time horizons. Both are clearly superior to the clockwise protocols. The clock3D protocol is moderately inferior to the clockIL protocol, but far superior to the clock2D protocol.

Quantitative results are summarised in Figure 13 and Table 3. For each protocol, Figure 13 plots the relative area $A(t) / A_{0}$ of $\mathcal{S}(t)$ on a semilog scale against the number $t / T$ of elapsed stirring periods. The empirical entropy of each protocol is determined by fitting a straight line to (the upper portion of) the corresponding area curve. 
All protocols except clock2D clearly have nonzero entropies. In terms of full period entropy $h=\log \lambda$, the best protocol is pig3D, followed respectively by pigIL and clockIL. However, the ranking of Table 3 is based on entropy per generator or entropy rate $\mathrm{h} / \mathrm{T}$, a more meaningful measure from the viewpoint of energy efficiency. On this basis the favourite is the pig2D protocol, which surpasses the theoretical entropy rate of 0.481 . This benchmark rate is also achieved by the pig3D and pigIL protocols, albeit only after three to four periods. Contrary to (tentative) theoretical predictions, the clock3D and clockIL protocols appear asymptotically equivalent and uniformly inferior to their pigtail counterparts. However, the erratic character of the clock3D growth curve indicates persistence of the 'snap-back' phenomenon and suggests qualitative inferiority to the clockIL protocol. Intriguingly, the (joint) asymptotic efficiency of the clock3D and clockIL protocols appears to match the clock3D theoretical upper bound of $h=\log 4 \approx 1.39$.

\subsection{Discussion}

Our theoretical and numerical results are in good qualitative agreement and partial quantitative agreement. At a minimum, our results suggest that three dimensionality strongly enhances the efficiency of topologically trivial two dimensional protocols - a conjecture with promising implications for lid driven cavity flows. Conversely, three dimensionality appears to slightly degrade the quantitative performance of topologically complex protocols.

From a practical perspective, it is highly inadvisable to rely on a single metric of mixing efficiency. The pig2D protocol provides an extreme example: notwithstanding its outstanding entropy rate, it induces no lateral mixing at all. In any event, the artificial nature of our hypothetical batch mixer must be borne in mind. For example, its peculiar combination of slip and no-slip boundaries arguably penalises three dimensionality and privileges the two dimensional protocols (via invariant no-slip conditions on the end walls located at $z= \pm 1$ ). 
Given the necessarily tentative nature of our conclusions, we are undertaking enhancements in several directions. At the theoretical level, we are seeking more robust entropy estimates, analogous to the Moussafir [8] formulae for $2 \mathrm{D}$ braids. Qualitatively, we are applying alternative metrics such as lateral mixing, shape (of particle trajectories and advected area filaments) and degree of homogenisation (estimated via advection of particle clusters). Quantitatively, we are improving the meshing algorithm and expanding the regimen of convergence testing. Ideally, each quantitative simulation should be run as long as possible in order to eliminate transient effects. However, mesh quality inevitably degrades over time, and no amount of mesh refinement will maintain a uniformly smooth representation of a large surface $\mathcal{S}(t)$ comprising hundreds or even thousands of folds.

Last but not least, we are applying 3D braiding theory to more realistic mixing devices. Figures 14 and 15 present simulations for one such prototypical device, comprising a rectangular cavity fitted with an asymmetric roller lid at the top face of the cavity. The fluid is again assumed to be inertialess; however, unlike the hypothetical mixer of Section 3.1, it is modelled with no-slip boundary conditions on all six cavity walls. (Vertical symmetry is now absent, but could be restored by introducing a second moving lid on the bottom face of the cavity.) The lid comprises two sections of unequal size, moving in opposite directions to induce vortical motion of the interior fluid. Motion in the $x$-direction simulates a $\sigma$ operation; conversely, $z$-wise motion creates a $\Sigma$ operation. In this way, any protocol in Table 1 can be simulated. Our aim is to demonstrate that these lid driven mixing protocols are significantly more effective than conventional lid driven protocols of comparable energy cost, thereby reinforcing the importance of both topology and dimensionality to non-inertial fluid mixing.

Acknowledgements This work was supported by the ARC Discovery Grant DP0881054. 
clock3D
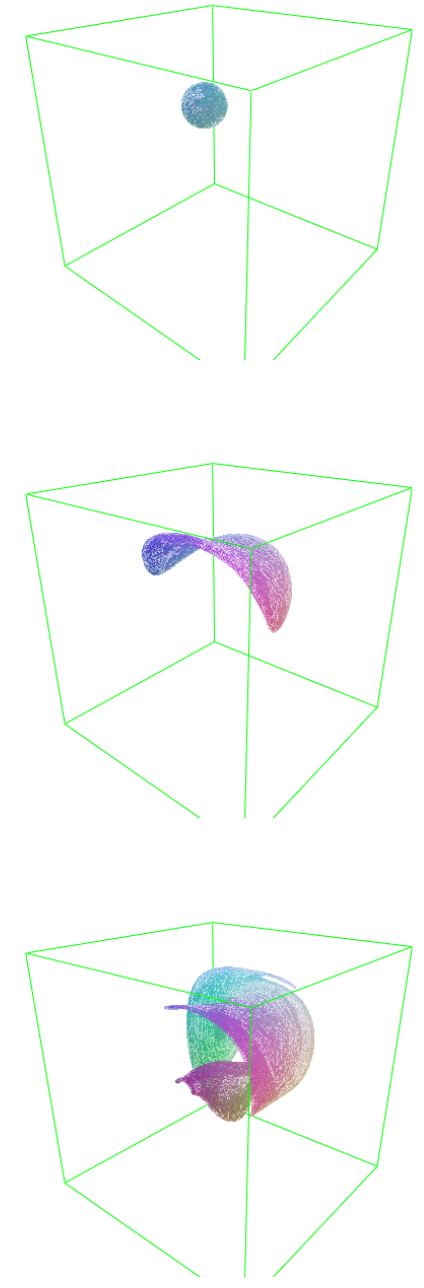

pig3D

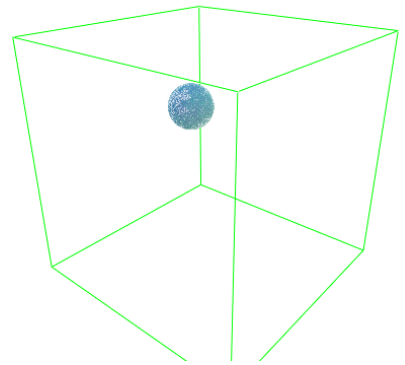

$$
t=0
$$

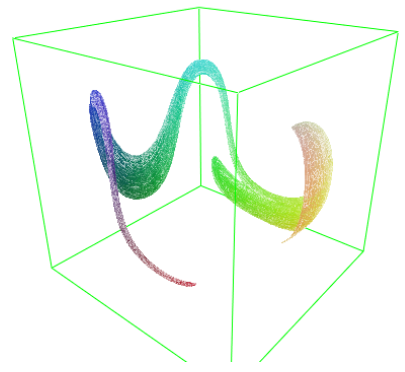

$$
\mathrm{t}=4
$$

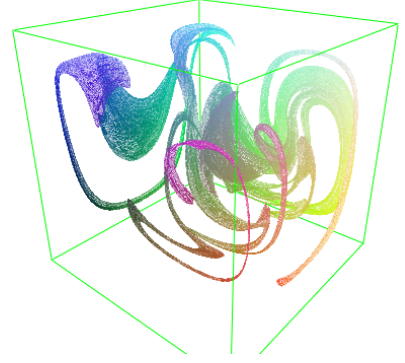

$$
t=8
$$

pigIL
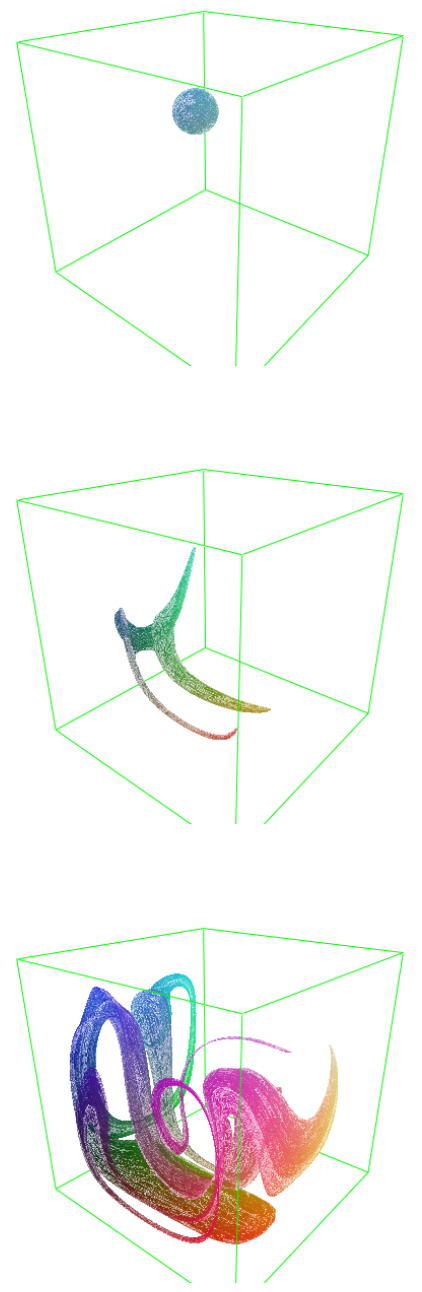

Figure 11: Advection of a bubble-like initial surface $\mathcal{S}_{0}=\mathcal{B}$ by two periods $(\mathrm{t}=0,4,8)$ of the clock3D, pig3D and pigIL protocols. The 'bubble' $\mathcal{B}$ is an off-centre sphere of radius $r=0.2$, corresponding to one-fifth of the cavity half-width. 


\section{$\mathrm{t}=12$}

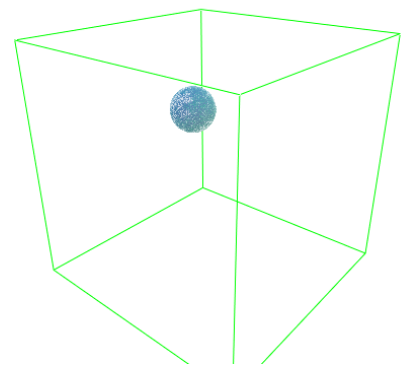

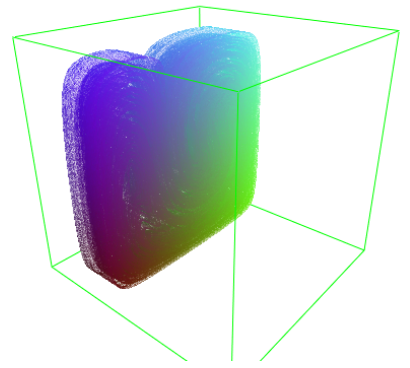

pig2D

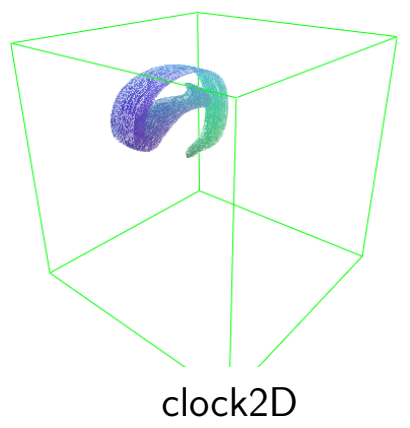

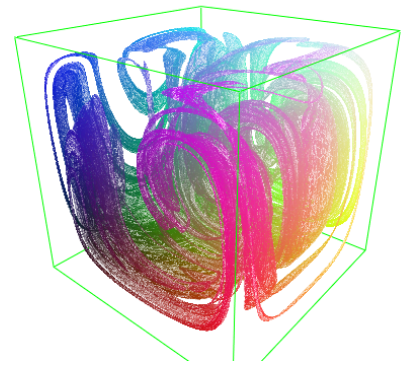

pig3D

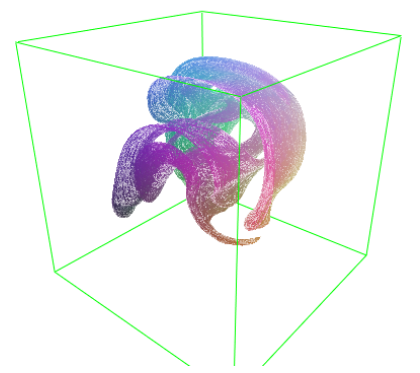

clock3D

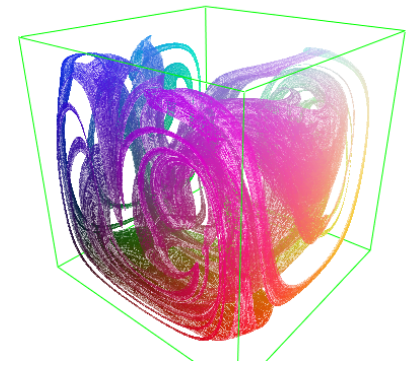

pigIL

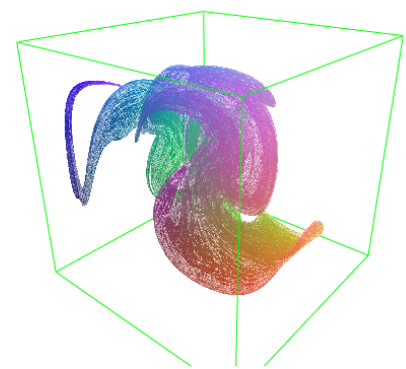

clockIL

Figure 12: Advection of the bubble-like initial surface $\mathcal{S}_{0}=\mathcal{B}$ under 12 units of each of the six protocols as indicated. 

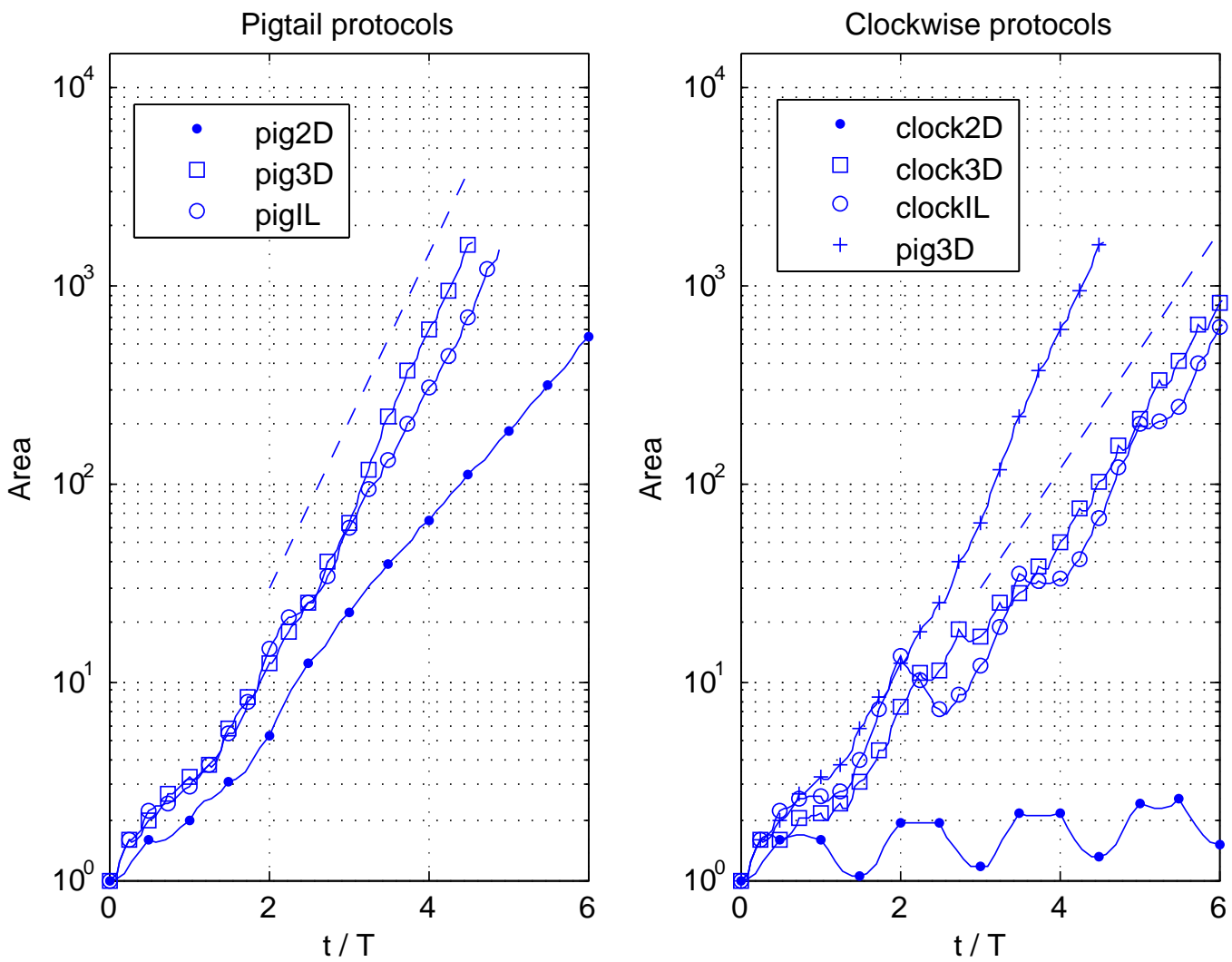

Figure 13: Semilog plots of relative area $A(t) / A_{0}$ (with initial surface $\mathcal{S}_{0}=\mathcal{B}$ ) for each protocol, plotted against the number $t / T$ of elapsed stirring periods. The marker spacing is $\Delta t=1$ throughout, corresponding to one full phase of each protocol. The dashed line at left indicates the asymptotic growth rate $A(t)=\mathcal{O}\left((2.618)^{2 t / T}\right)$ of the pigtail protocols; the dashed line at right indicates the optimistic estimate $\mathcal{O}\left(2^{(2 t / T)}\right)$ for the clock3D protocol. The pig3D data is reproduced at right for comparison purposes. 
pig2D
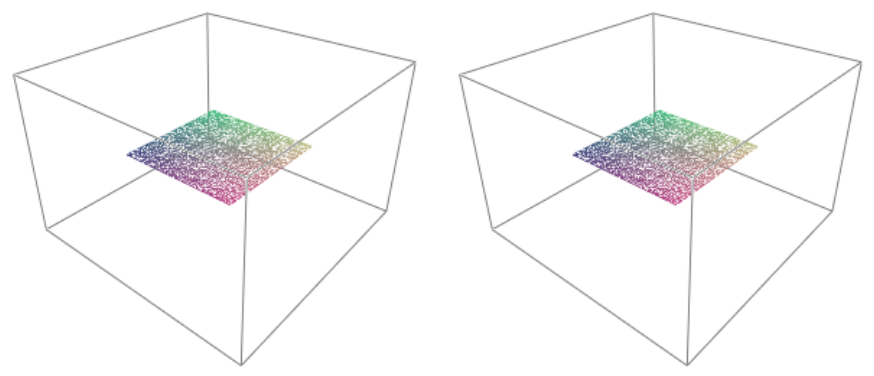

$\mathrm{t}=0$
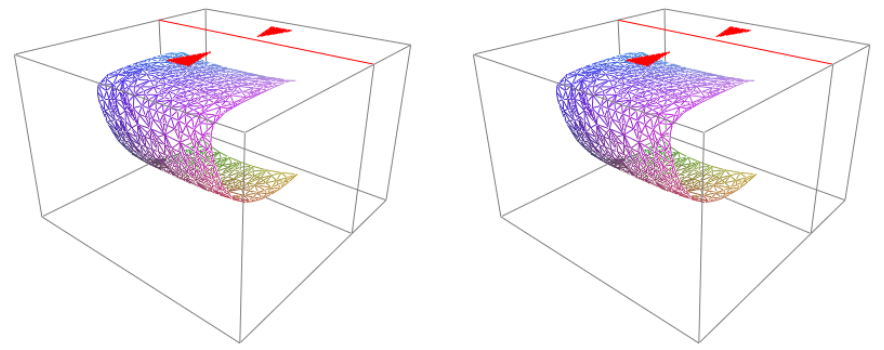

$$
t=1
$$
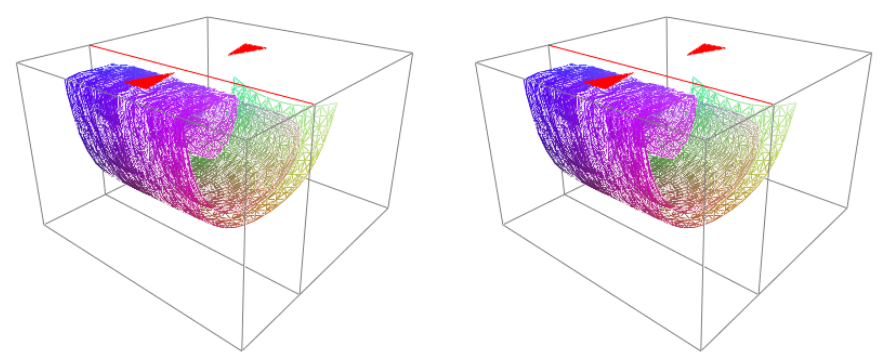

$$
\mathrm{t}=2
$$

pigIL
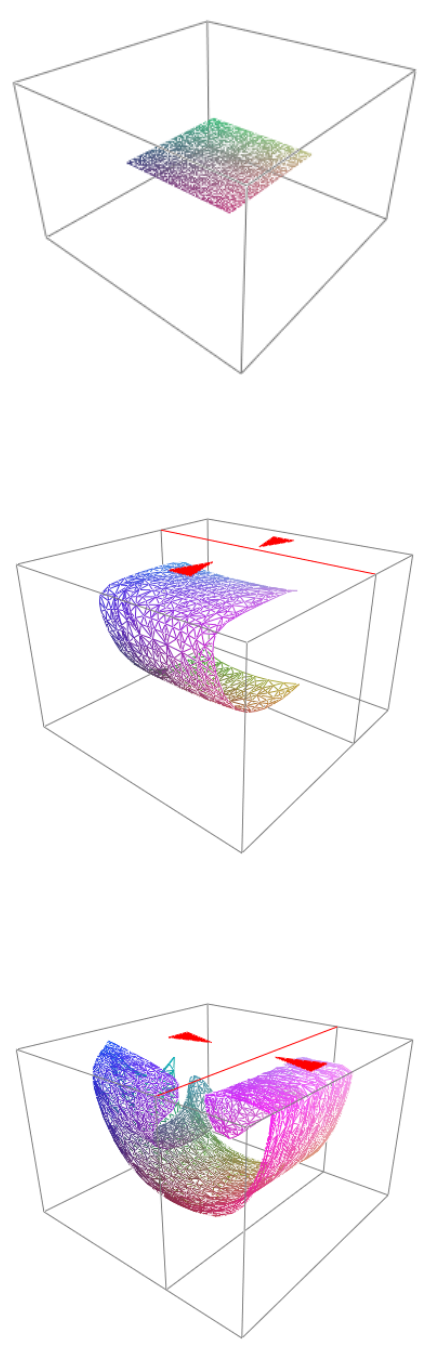

Figure 14: Implementation of the pigtail protocols (shown for $t=0,1,2$ ) by an alternating lid driven cavity flow. The cavity is rectangular, with rigid side and bottom walls. The top wall comprises two asymmetric roller lids, whose motion (indicated by red arrows) induces vortical flow within the cavity interior. 
pig2D
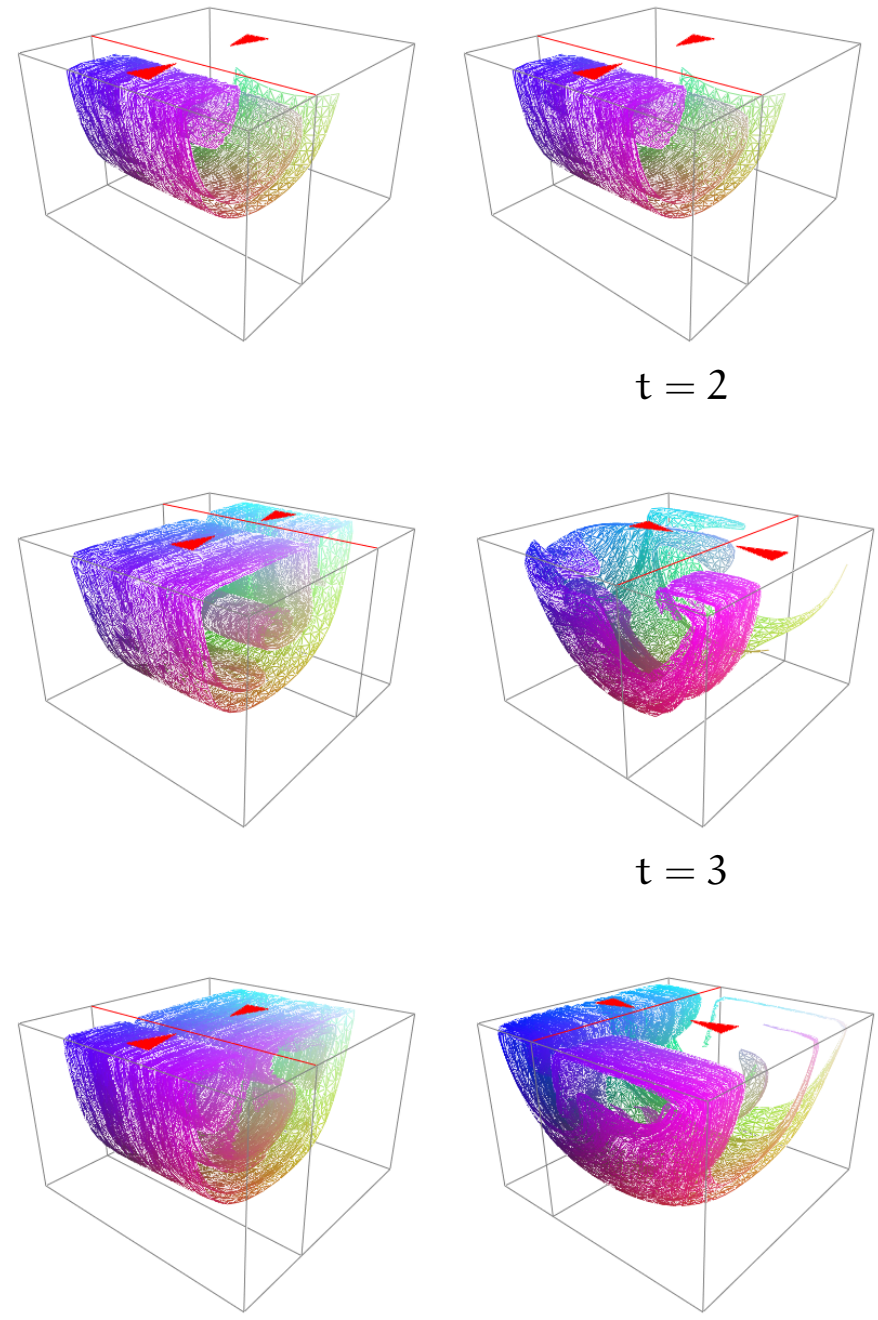

$$
\mathrm{t}=4
$$

pigIL
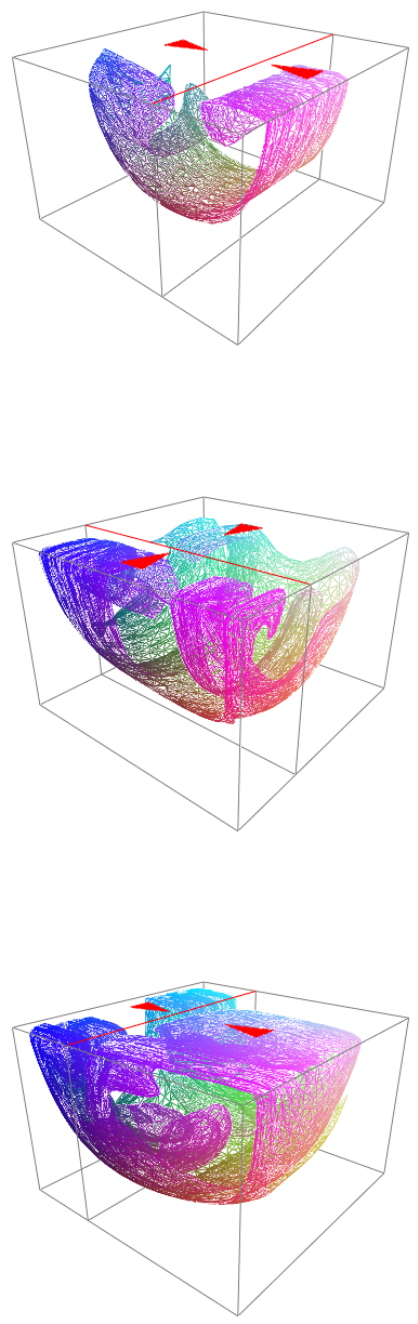

Figure 15: Cavity flow implementation of the pigtail protocols $(t=2,3,4)$. 


\section{References}

[1] M. Bestvina and M. Handel. Train tracks for surface homeomorphisms, Topology 34, 1995, 109-140. doi:10.1016/0040-9383(94)E0009-9 C882

[2] P. L. Boyland, H. Aref and M. A. Stremler. Topological fluid mechanics of stirring, J. Fluid Mech., 403, 2000, 277-304. doi:10.1017/S0022112099007107 C876, C880, C881, C883

[3] J. Chen and M. A. Stremler. Topological chaos and mixing in three dimensional channel flow. Physics of Fluids, 21, 2009, 021701. doi:10.1063/1.3076247 C883

[4] M. D. Finn and J.-L. Thiffeault. Topological entropy of braids on the torus. SIAM J. Appl. Dyn. Sys., 6, 2007, 79-98. doi:10.1137/060659636 C882

[5] O. S. Galaktionov, P. D. Anderson, G. M. W. Peters and F. N. van de Vosse. An adaptive front tracking technique for three dimensional transient flows. Int. J. Numer. Meth. Fluids, 32, 2000, 201-217. C899

[6] E. Gouillart, N. Kuncio, O. Dauchot, B. Dubrulle, S. Roux and J.-L. Thiffeault. Walls inhibit chaotic mixing. Phys. Rev. Lett. 99, 2007, 114501. C883

[7] T. G. Kang, M. K. Singh, T. H. Kwon and P. D. Anderson. Chaotic mixing using periodic and aperiodic sequences of mixing protocols in a micromixer. Microfluid Nanofluid, 4, 2008, 589-99. doi:10.1007/s10404-007-0004-x C883, C892

[8] J.-O. Moussafir. On computing the entropy of braids. Func. Anal. Other Math., 1, 2006, 37-46. doi:10.1007/s11853-007-0206-z C882, C902

[9] H. A. Stone and S. Kim. Microfluidics: basic issues, applications and challenges. American Institute of Chemical Engineers Journal, 47, 2001, 1250-54. doi:10.1002/aic.690470602 C876 
[10] M. A. Stremler and J. Chen. Generating topological chaos in lid driven cavity flow, Physics of Fluids, 19, 2007, 103602. doi:10.1063/102772881 C883, C892

[11] M. A. Stremler, F. R. Haselton and H. Aref. Designing for chaos: applications of chaotic advection at the microscale. Phil. Trans. R. Soc. Lond. A, 362, 2004, 1019-36. C876

[12] J.-L. Thiffeault and M. D. Finn. Topology, braids and mixing in fluids. Phil. Trans. R. Soc. Lond. A, 364, 2006, 3251-66. doi:10.1098/rsta.2006.1899 C880, C881, C885

[13] C. R. Thomas. Problems of shear in biotechnology. Critical Reports on Applied Chemistry, 29, 1990, 23-93. C876

[14] S. O. Unverdi and G. Tryggvason. A front tracking method for viscous, incompressible, multi-fluid fluids. J. Comp. Phys., 100, 1992, 25-37. C899

[15] G. M. Whitesides and A. D. Stroock. Flexible methods for microfluidics. Physics Today, 54, 2001, 42-48. C876, C892

\section{Author address}

1. N. D. Jewell, School of Mathematical Sciences, The University of Adelaide, Australia.

mailto:nathaniel.jewell@adelaide.edu.au 